\title{
Hyperactivation of JAK1 tyrosine kinase induces stepwise, progressive pruritic dermatitis
}

\author{
Takuwa Yasuda, ${ }^{1}$ Toshiyuki Fukada, ${ }^{2,3}$ Keigo Nishida, ${ }^{2,4}$ Manabu Nakayama, ${ }^{5}$ Masashi Matsuda, ${ }^{6}$ Ikuo Miura, ${ }^{7}$ Teruki Dainichi, ${ }^{8}$ \\ Shinji Fukuda, ${ }^{9,10}$ Kenji Kabashima, ${ }^{8}$ Shinji Nakaoka, ${ }^{11,12}$ Bum-Ho Bin, ${ }^{13}$ Masato Kubo, ${ }^{14}$ Hiroshi Ohno, ${ }^{9}$ Takanori Hasegawa, ${ }^{6}$ \\ Osamu Ohara, ${ }^{5,15}$ Haruhiko Koseki, ${ }^{6}$ Shigeharu Wakana,7 and Hisahiro Yoshida'
}

\begin{abstract}
'Laboratory for Immunogenetics and 'Laboratory for Cytokine Signaling, RIKEN Research Center for Integrative Medical Sciences (IMS-RCAI), Yokohama, Japan. ${ }^{3}$ Tokushima Bunri University, Faculty of Pharmaceutical Sciences, Molecular and Cellular Physiology, Tokushima, Japan. ㄴaboratory of Immune Regulation, Faculty of Pharmaceutical Science, Suzuka University of Medical Science, Mie, Japan. 5Department of Technology Development, Kazusa DNA Research Institute, Kisarazu, Japan. ' ${ }^{2}$ aboratory for Developmental Genetics, RIKEN Research Center for Integrative Medical Sciences (IMS-RCAI), Yokohama, Japan. ${ }^{7}$ Technology and Development Team for Mouse Phenotype Analysis: Japan Mouse Clinic, RIKEN BioResource Center, Tsukuba, Japan. ${ }^{8}$ Department of Dermatology, Kyoto University Graduate School of Medicine, Kyoto, Japan. ${ }^{9}$ Laboratory for Intestinal Ecosystem, RIKEN Research Center for Integrative Medical Sciences (IMS-RCAI), Yokohama, Japan. ${ }^{10}$ Institute for Advanced Biosciences, Keio University, Yamagata, Japan. "'Laboratory for Mathematical Modeling of the Immune System, RIKEN Research Center for Integrative Medical Sciences (IMS-RCAI), Yokohama, Japan. ${ }^{12}$ Department of Global Health Policy, Graduate School of Medicine, The University of Tokyo, Tokyo, Japan. ${ }^{3 B}$ Bioscience Research Institute, Amorepacific Corporation R\&D Center, Yongin, South Korea. ${ }^{14}$ Laboratory for Cytokine Regulation and ${ }^{15}$ Laboratory for Integrative Genomics, RIKEN Research Center for Integrative Medical Sciences (IMS-RCAI), Yokohama, Japan.

Skin homeostasis is maintained by the continuous proliferation and differentiation of epidermal cells. The skin forms a strong but flexible barrier against microorganisms as well as physical and chemical insults; however, the physiological mechanisms that maintain this barrier are not fully understood. Here, we have described a mutant mouse that spontaneously develops pruritic dermatitis as the result of an initial defect in skin homeostasis that is followed by induction of a Th2-biased immune response. These mice harbor a mutation that results in a single aa substitution in the JAK1 tyrosine kinase that results in hyperactivation, thereby leading to skin serine protease overexpression and disruption of skin barrier function. Accordingly, treatment with an ointment to maintain normal skin barrier function protected mutant mice from dermatitis onset. Pharmacological inhibition of JAK1 also delayed disease onset. Together, these findings indicate that JAK1-mediated signaling cascades in skin regulate the expression of proteases associated with the maintenance of skin barrier function and demonstrate that perturbation of these pathways can lead to the development of spontaneous pruritic dermatitis.
\end{abstract}

\section{Introduction}

The skin provides a strong and flexible barrier that protects the host from foreign particles and microbial invasion and maintains homeostatic water loss from the body's surface. Since it is continuously damaged physically and chemically by the environment, this skin barrier system, the stratum corneum in the outermost layer, must be maintained and continuously regenerated by the proliferation and differentiation of keratinocytes. If this skin barrier system is disrupted, various skin diseases such as ichthiosis vulgaris and atopic dermatitis can result (1), although a skin immune reaction is also a requisite for the development of such inflammatory conditions. Skin homeostasis is thought to be controlled by a balance of various factors present in the skin tissue that allows for flexibility in dealing with a changing environment (2). For example, epidermal growth factor family members and their receptors are known to control the differentiation and development of epidermal cells to maintain a normal epidermal component $(3,4)$; proinflammatory cytokines such as IL-6 and IL-1 $\beta$ play important roles in wound healing and regeneration of epidermal tissue $(5,6)$; and several cytokines are important for keratinocyte proliferation and differentiation in inflammatory disease

Conflict of interest: The authors have declared that no conflict of interest exists. Submitted: May 29, 2015; Accepted: March 3, 2016.

Reference information: J Clin Invest. 2016;126(6):2064-2076. doi:10.1172/JCI82887. conditions such as psoriasis $(7,8)$. However, the precise signal transduction molecules activated by these cytokines and their receptors in skin barrier maintenance are not fully understood.

In the present study, we identified a skin disease animal model through phenotypic screening of $\mathrm{N}$-ethyl $\mathrm{N}$-nitorosourea-induced (ENU-induced) randomly mutagenized mice (9). The mutant mouse described here spontaneously developed dermatitis accompanied by scratching behavior and, as the disease progressed, immunological abnormalities that developed in a stepwise manner. We identified the responsible gene by genetic mapping, and by sequencing the candidate gene Jak1, we identified a gain-of-function missense point mutation in the coding region.

JAK1 is a signal transduction molecule associated with various cytokine receptors that participates in several signaling pathways to activate other molecules such as STATs or GRB2 (10). More than 10 cytokines are known to be ligands of JAK1-associated receptors and have activities on various cells and tissues including leukocytes, neurons, and epithelial cells (11). By means of genetic analysis and BM transfer experiments, we found that the Jak1 point mutation induces spontaneous skin barrier disruption and that skin is the responsible tissue for dermatitis onset. We discovered that several serine proteases regulated by the JAK1 signaling pathway are overexpressed in mutant skin and downregulated with JAK inhibitor administration. 
In addition, we found that emollient treatment effectively delays the onset of dermatitis in this model.

Here, we describe a unique dermatitis model that mimics human disease in terms of genetic factors, that of a single gene mutation affecting both skin barrier homeostasis and immune responses, and in terms of an environmental factor regulating disease onset.

\section{Results}

Identification and characterization of the dermatitis model. Dermatitis in this mutant mouse manifests as redness and desquamation of the ears due to scratching and occurs in specific pathogen-free (SPF) conditions in an autosomal recessive manner (Figure 1A and Supplemental Video 1; supplemental material available online with this article; doi:10.1172/JCI82887DS1). In adult homozygotes, skin lesions are characterized by epidermal hyperplasia and the infiltration of mononuclear inflammatory cells including mast cells, eosinophils, and $\mathrm{CD} 4^{+} \mathrm{T}$ cells (Figure $1 \mathrm{~B}$ and Supplemental Figure 1). Dermatitis penetrance diagnosed by Matsuda's clinical criteria (12) reaches $100 \%$ in homozygotes by the time they reach 12 weeks of age (Figure 1, C and D). Starting at approximately 3 weeks after the onset of dermatitis, serum IgE and IgG1 Ab levels progressively increased with age (Figure 1, E and F), and by 12 to 14 weeks, serum histamine levels were also elevated (Figure 1G). From approximately 8 weeks after the onset of dermatitis, serum IgG2b and IgG2c levels were also elevated (Supplemental Figure 2). To further examine the Th2 and Th1 immune responses in dermatitis-afflicted homozygotes, we measured cytokine production by $\mathrm{CD} 4^{+} \mathrm{T}$ cells. IL-4, IL-5, and IL-13 levels were significantly higher than those in WT mice at 10 and 20 weeks of age, whereas the production of IFN- $\gamma$ in homozygotes was augmented, but not until 20 weeks of age (Supplemental Figure 3). These results suggest that the Th2related immune responses in homozygotes occur a few weeks after the onset of dermatitis and that chronic inflammatory conditions ultimately result in both Th2 and Th1 immune responses. A small fraction $(<20 \%)$ of heterozygotes also developed mild dermatitis, but it was not detected until after 6 months of age (Supplemental Figure 4). Given its phenotype, we named this mutant mouse strain Spade (stepwise, progressive atopic dermatitis).

Detection of the causative Spade mutation in the Jak1 gene. To define the causative dermatitis gene in the mutant mice, we performed genetic mapping of the Spade allele in the C57BL/6J (B6) strain by outbreeding with the $\mathrm{C} 3 \mathrm{H} / \mathrm{HeJ}$ (C3) strain (13) and located it on chromosome 4 (Supplemental Figure 5). Further screening for candidate genes identified a G-to-A missense mutation at position 2633 in Jak1 in Spade homozygotes that resulted in an arginine (R) to histidine $(\mathrm{H})$ substitution at position 878 in the protein, located near the ATP-binding motif of the tyrosine kinase domain (Figure 2, A and B). To confirm that this Jak1 point mutation was sufficient to drive the development of dermatitis in homozygotes, we generated a Jak1 knockin allele harboring the Spade point mutation (Figure 2C). Homozygous knockin mice developed spontaneous progressive dermatitis that phenocopied the disease in homozygous Spade mice (Figure 2, D-I). Thus, a single nucleotide mutation in Jak1 resulting in the $\mathrm{R} 878 \mathrm{H}$ substitution is linked to the Spade phenotype. We designated this allele Jak1 ${ }^{\text {Spade }}$ and will hereafter refer to this mutant as Spade.
The Spade mutation results in hyperactivation of the JAK1 tyrosine kinase. The JAK1 tyrosine kinase is a cytoplasmic protein that is activated through various cytokine receptors to initiate multiple signal transduction pathways (11). Since the Jak1 $1^{\text {Spade }}$ point mutation was found in the protein kinase domain and localized in the vicinity of the ATP-binding domain, we tested whether it affects JAK1 tyrosine kinase function. After JAK1-mediated phosphorylation, STAT proteins form dimers and translocate to the nucleus, where they activate gene expression (11). JAK1, STAT3, and STAT1 were constitutively hyperphosphorylated in Jak1 $1^{\text {Spade/spade }}$ mouse embryonic fibroblasts (MEFs) compared with that observed in WT MEFs, and the inducible phosphorylation of each of these molecules was further exaggerated upon stimulation with IL-6 or IFN- $\alpha$ (Figure 3, A-C). These results suggested that Spade is a gain-of-function mutation. In addition, expression levels of SOCS3 and SOCS1, transcriptionally activated molecules in the JAK/STAT pathway (10), were upregulated in Jak1 $1^{\text {Spade/spade }}$ MEFs compared with expression levels in WT MEFs after stimulation with IL-6 or IFN- $\alpha$ (Figure 3, D and E). Moreover, HA-tagged JAK $1^{\text {Spade }}$ that was expressed and activated in an in vitro system had higher catalytic activity against recombinant STAT3 than did WT JAK1 expressed in the same system (Figure 3F and ref. 14). These results clearly show that JAK $1^{\text {Spade }}$ is catalytically more active than WT JAK1 in terms of its phosphotyrosine kinase activity toward itself and STAT proteins. Since JAK1 is expressed in all cells and tissues $(11,15)$, we sought to determine the cellular origin of the dermatitis phenotype by characterizing the expression of phosphorylated pY-JAK1 in the ear skin of mutant and WT littermates. Phosphorylated JAK1 was detected in presymptomatic Jak1 $1^{\text {Spade/spade }}$ epidermis, but was scarcely detected in WT tissues (Figure 3G). This was accompanied by increased numbers of proliferating keratinocytes with Ki67 expression and ectopic expression of cytokeratin 6 (K6) in mutant skin (Figure 3G). Together, these results indicate that the proliferation and differentiation process is perturbed in the mutant keratinocytes.

In addition to tyrosine-phosphorylated JAK1 (pY-JAK1) (Figure 3G), pY-STAT1, -STAT2, -STAT3, -STAT5, and -STAT6 were present in the skin epidermal layer of 6-week-old Jak1 $1^{\text {spade/spade }}$, but not WT, mice (Supplemental Figure 6A). Moreover, the phosphorylation of these STATs was augmented by peritoneal administration of IL-6 (Supplemental Figure 6B). These data suggest that an intrinsic perturbation in the signaling interaction disrupted keratinocyte development in Jak1 $1^{\text {Spade/spade }}$ mice.

Skin is the tissue responsible for dermatitis onset, but immune cells can modulate dermatitis symptoms. The results in Figure 3 and Supplemental Figure 6 summarize the evidence that the incidence of dermatitis is associated with aberrant JAK1 kinase activity in situ. Because JAK1 is expressed in many cell types, including immune cells, we asked whether hematopoietic lineage cells also participate in the induction of dermatitis as a consequence of the expression of mutant Jak1. To this end, we generated Jak1 $1^{\text {Spade/Spade }}$ mice that lacked $\mathrm{T}$ and $\mathrm{B}$ cells by crossing them with $\mathrm{Rag1}^{-/-}(16)$ mice.

As shown in Figure 4, A-C, histological analysis revealed that the incidence, kinetics, and penetrance of dermatitis were similar in Jak1 $1^{\text {Spade/spade }}$ and Rag1/- Jak1 $1^{\text {Spade/Spade }}$ mice. However, the clinical dermatitis scores were significantly lower in the Jak1 $1^{\text {Spade/Spade }}$ mice with no B or T cells ( $\left.\mathrm{Rag1}^{-/-} \mathrm{Jak1} 1^{\text {Spade/Spade }}\right)$, indicating that lymphoid 
A

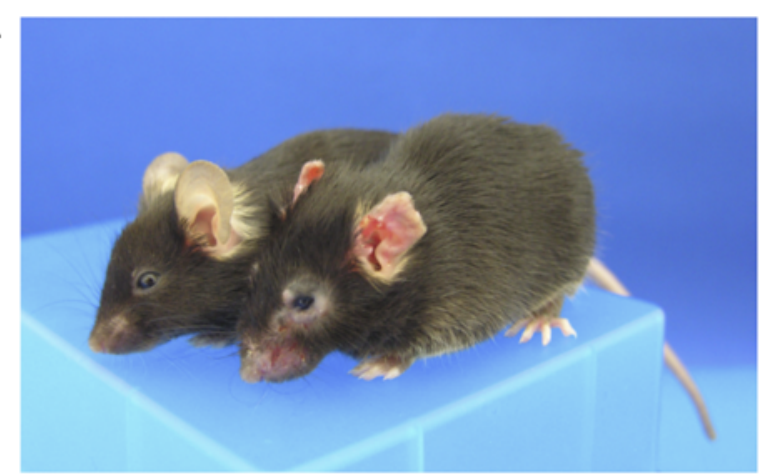

B
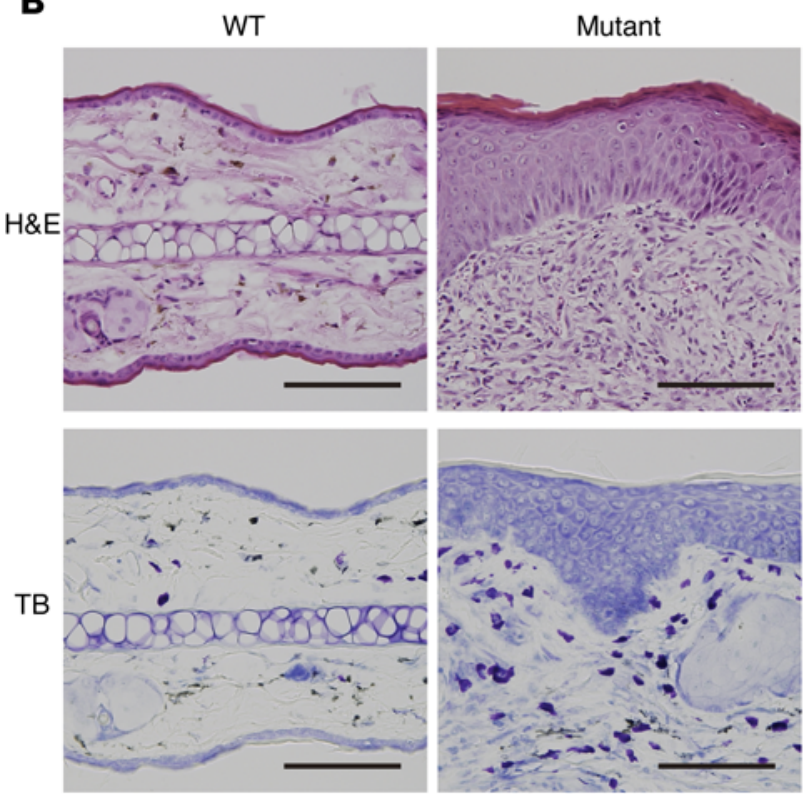

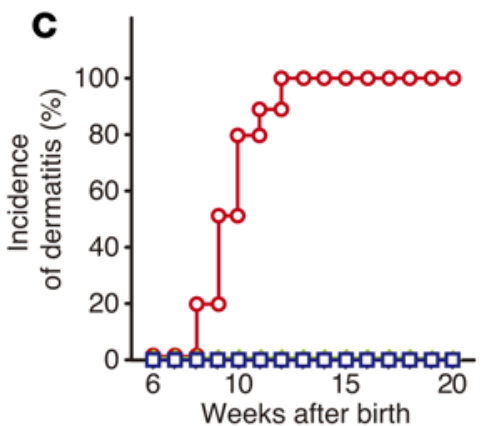

$\mathbf{E}$

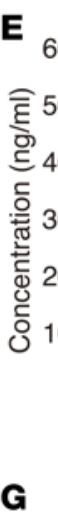

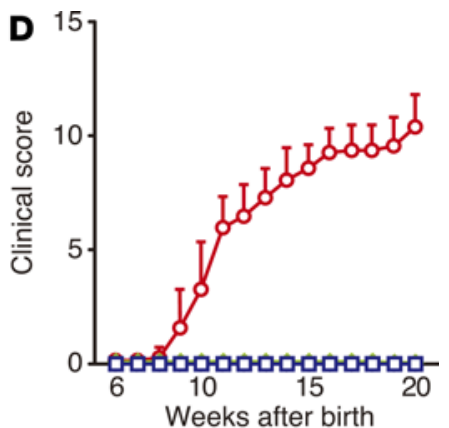
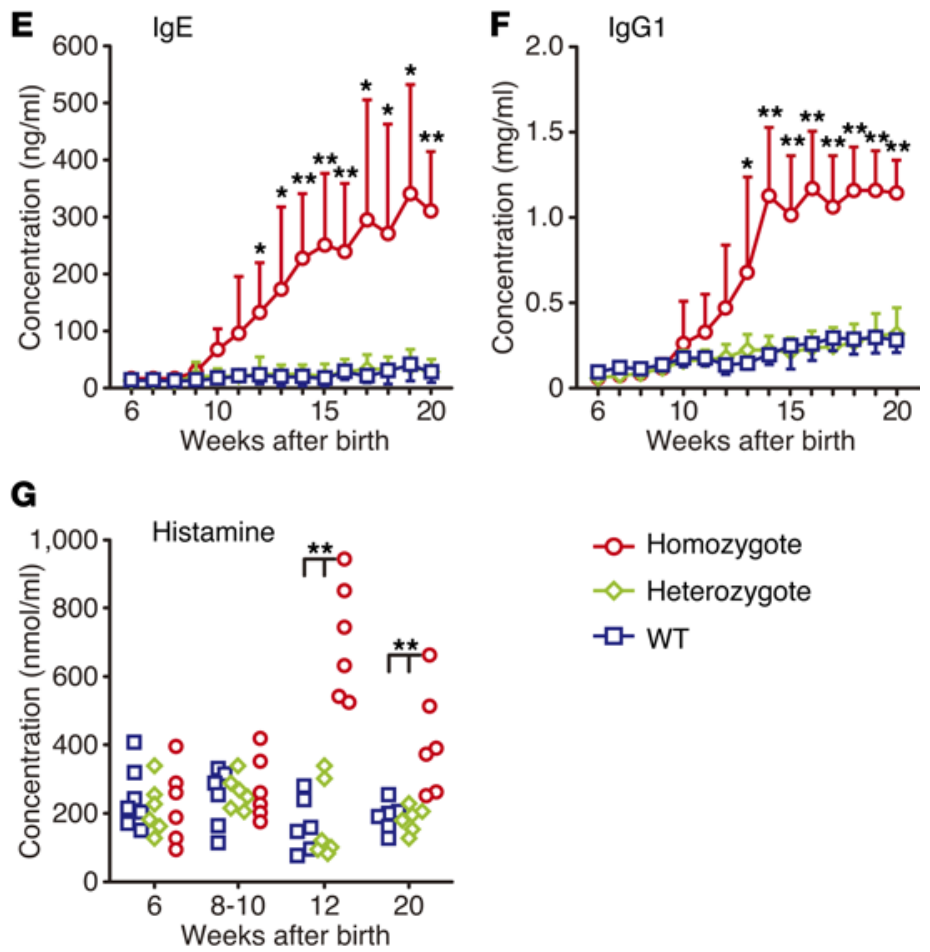

$$
\begin{aligned}
& -\sim \text { Homozygote } \\
& \prec \text { Heterozygote } \\
& -\square-\text { WT }
\end{aligned}
$$

Figure 1. Phenotypic characterization of dermatitis induced by ENU mutagenesis. (A) A mutant mouse (right) and a WT littermate (left) at 20 weeks of age. (B) Photomicrographs of histological sections of ear skin from mutant and WT mice (18 weeks of age) stained with H\&E or TB. Scale bars: $100 \mu \mathrm{m}$. (C and D) Longitudinal analyses of dermatitis incidence (C) and clinical scores (D) of homozygous (red), heterozygous (green), and WT (blue) mice. The cumulative incidence of dermatitis plotted in panel $\mathbf{C}$ was determined from cohorts of 45 mutant homozygous, 20 heterozygous, and 50 WT mice. (E and F) Longitudinal analyses of levels of serum IgE (E) and IgG1 (F) in homozygous, heterozygous, and WT mice. (G) Longitudinal analyses of serum histamine levels in homozygous, heterozygous, and WT mice. Data in D-F represent the mean \pm SD of 8 mice per group. ${ }^{*} P<0.05$ and ${ }^{*} P<0.01$, relative to both heterozygous and WT mice, by 1-way ANOVA, followed by Bonferroni's test.

cells can exacerbate the dermatitis symptoms (Figure 4, A-C). In fact, the proliferation of thymocytes in response to various cytokines was higher in Spade-mutant mice than in their WT littermates (Supplemental Figure 7).

Considering that mast cells play a crucial role in skin dermatitis (17), we examined their possible involvement by crossing Jak1 $1^{\text {Spade/Spade }}$-mutant mice with Kit ${ }^{W-s h / W-s h}(18)$ mice. As shown in Figure 4, D-F, the congenital lack of mast cells did not significantly influence the development of dermatitis in Jak1 $1^{\text {pade/spade }}$ mice.

To assess the role of any other hematopoietic or nonhematopoietic cells in the dermatitis, we generated BM chimeras using WT and Jak1 $1^{\text {Spade//pade }}$ mice. Jak1 $1^{\text {spade/spade }}$ hosts harboring WT BM cells developed dermatitis 10 weeks after transfer, whereas chimeras with the reciprocal combination of BM did not develop the disease, even 6 months after transfer (Figure 5A). Although expression of the Jak1 $1^{\text {spade }}$ allele by BM-derived cells alone was insufficient to drive dermatitis, epidermal hyperplasia and inflammatory cell infiltration were more rapid and severe in $J a k 1^{\text {Spade/spade }} \mathrm{BM} \rightarrow J a k 1^{\text {Spade/spade }}$ chimeras than in WT BM $\rightarrow J a k 1^{\text {Spade/spade }}$ chimeras (Figure 5, B and C). Together, these results demonstrate that the development of dermatitis in Jak1 $1^{\text {spade/spade }}$ mice requires expression of the Jak1 $1^{\text {spade }}$ allele by nonhematopoietic cells but that the expression of this allele in the hematopoietic compartment exacerbates the disease severity.

Permeability barrier function is defective in Spade-mutant skin. Skin barrier defects are known to cause ichthyosis and atopic dermatitis (2). To investigate whether dermatitis in $\mathrm{Jak1}^{\text {Spade/spade }}$ mutant mice is associated with aberrant epidermal barrier 
A

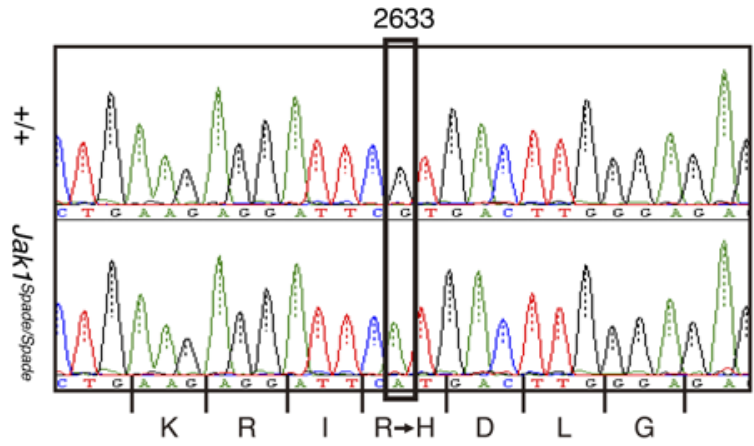

B

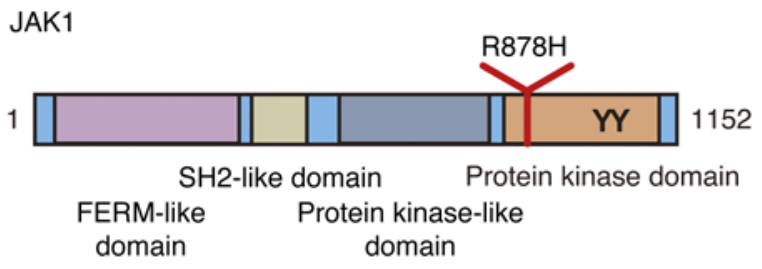

C

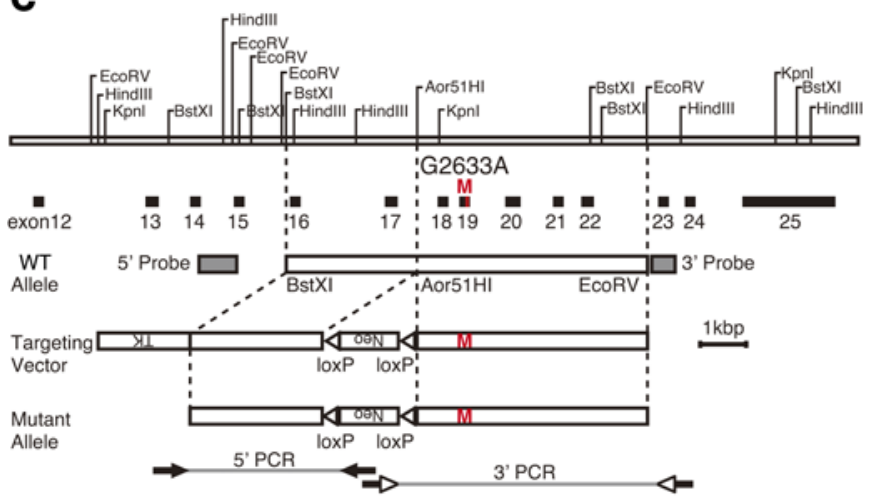

$\mathbf{F}$

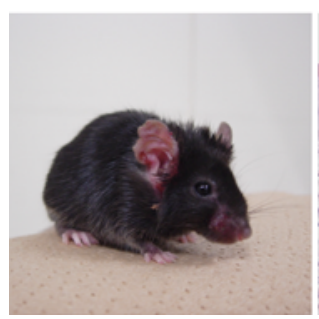

G

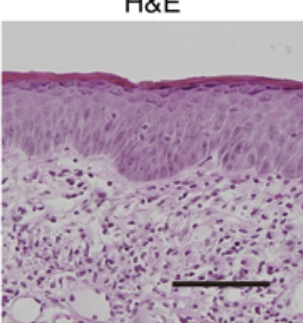

TB

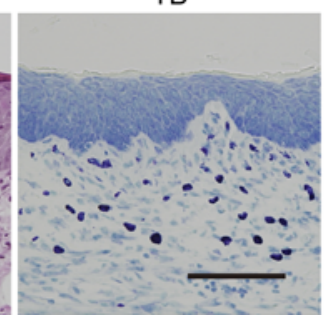

D

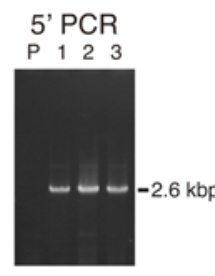

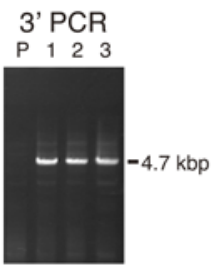

E

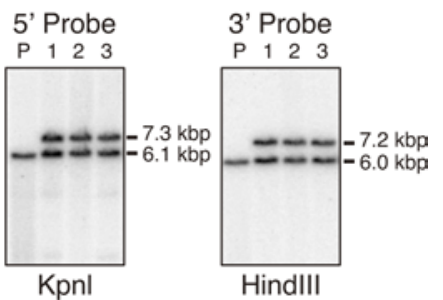

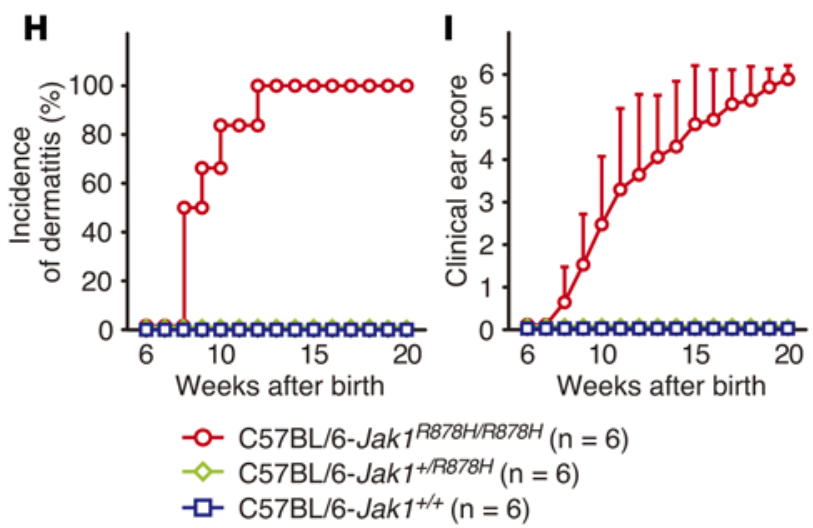

Figure 2. Dermatitis is caused by a missense mutation in the Jak1 tyrosine kinase expressed in nonhematopoietic cells. (A and B) Detection of a Jak1 G-to-A point mutation in Jak $7^{\text {Spade/Spade }}$-mutant mice (A) that replaces an arginine with a histidine at position 878 in the protein kinase domain of JAK1 (B). FERM, 4.1, ezrin, radixin, moesin; SH2, Src homology region 2. (C) Diagram of the Jak ${ }^{\mathrm{R} 878 \mathrm{H}}$ mutation knockin construct showing the location of the point mutation (red $\mathbf{M}$ in exon 19) and the selection cassette. (D) PCR amplification with the primers shown at the bottom of $\mathbf{C}$ was used to screen for homologous recombination in ES cells. (E) Southern blot of ES cell genomic DNA using the $5^{\prime}$ and $3^{\prime}$ probes depicted in panel $\mathbf{C}$, confirming homologous recombination. (F) Physical appearance of a homozygous knockin mouse. (C) Photomicrographs showing histological sections of H\&E- and TB-stained ear skin from a 20-week-old homozygous knockin mouse. Scale bars: $100 \mu \mathrm{m}$. Longitudinal analyses of dermatitis incidence (H) and clinical scores (I) of homozygous, heterozygous, and WT mice.

function, we tested the rate of transepidermal water loss (TEWL) as a readout for barrier function (19). The TEWL rate for skin from 6-week-old (presymptomatic) Jak1 $1^{\text {spade/spade }}$ mice was higher than that of skin from age-matched heterozygous and WT mice and became further elevated after dermatitis onset (Figure 6A). Biotin particles painted onto the ear skin of 6-week-old Jak1 $1^{\text {Spade/spade }}$ mice penetrated deeply into the stratum corneum, whereas these particles remained on the skin surface of WT mice (Figure 6B). Together, these data indicate that the physiological skin barrier of Jak $1^{\text {Spade/Spade }}$ mice is defective.

To investigate whether compromised skin barrier function plays a functional role in dermatitis development, we coated the ear skin of Jak1 $1^{\text {Spade/spade }}$ mice with petrolatum 3 times per week starting at 4 weeks of age. This treatment delayed the onset of dermatitis, and by 14 weeks of age, only $25 \%$ of the petrolatum-treated Jak1 $1^{\text {spade/spade }}$ mice had developed disease (Figure 6C). Dermatitis was also clinically less severe in petrolatum-treated mice (Figure 6D). Consistently, the TEWL rate of the ear skin remained low in petrolatum-treated Jak1 $1^{\text {Spade/spade }}$ mice that were protected from dermatitis (Figure 6E). By histopathological analysis, dermal infiltrations of neutrophils and monocytes were detected in the nontreated ears before disease onset but were suppressed in petrolatum-treated ears (Supplemental Figure 8). 

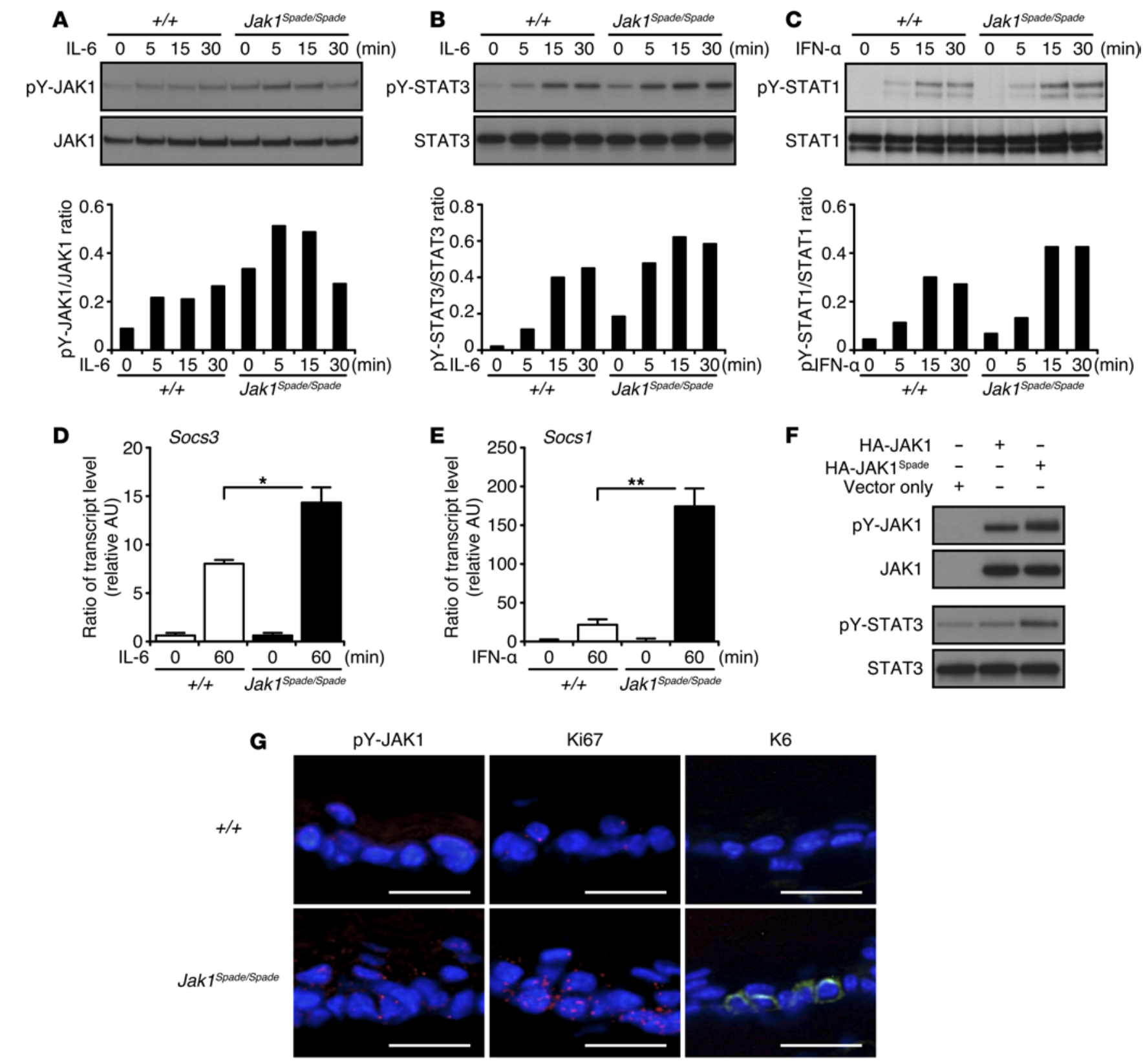

Figure 3. Constitutive activation of JAK1 promotes dermatitis in Jak15ade/spade mice. (A-C) Data are from immunoprecipitated lysates of MEFs stimulated with IL-6 (JAK1 and STAT3) or IFN- $\alpha$ (STAT1). Western blots and densitometric analysis showing cytokine-induced hyperphosphorylation of JAK1 (A), STAT3 (B), and STAT1 (C). (D and E) Cytokine-induced Socs3 (D) and Socs1 (E) transcript levels in Jak75pade/spade and WT MEFs stimulated with IL-6 or IFN- $\alpha$. Error bars represent the mean $\pm \mathrm{SD}$. ${ }^{*} P<0.05$ and ${ }^{* *} P<0.01$, by 2 -tailed Student's $t$ test. (F) Immunoblots showing in vitro phosphorylation of STAT3 by cytokine-activated JAK1 and JAK1 ${ }^{\text {Spade }}$ immunoprecipitated from 293T cells expressing a chimeric G-CSFR-gp130 receptor. (G) Frozen ear sections from Jak $7^{\text {Tpade/Spade }}$ and WT mice at 6 weeks of age were stained for pY-JAK1 and Ki67 (both red) and K6 (green), and nuclei were counterstained with DAPI (blue). Scale bars: $20 \mu \mathrm{m}$.

Next, to examine whether permeability barrier disruption has any significant effect on accelerating or worsening dermatitis in Spade-mutant mice, we disrupted the permeability barrier of Jak1 $1^{\text {spade/Spade }}, J a k 1^{+/ \text {Spade }}$, and WT mice using the sequential tape-stripping method described in reports by Feingold and Elias (20). In Jak1 $1^{\text {spade/spade }}$ mice, the onset of dermatitis was not accelerated by tape stripping, but in Jak1+/spade and WT mice, the treatment induced ear inflammation in both genotypes, although it was slightly more severe in $J a k 1^{+/ \text {spade }}$ mice. The inflammatory status, judged by histopathological findings, was comparable among all the mice (Supplemental Figure 9).

Overexpression of serine proteases in mutant skin induces the barrier defect. To define the genetic skin signature, we performed microarray analysis to compare the gene expression profiles of Jak1 $1^{\text {Spade/spade }}$ and WT ear skin at 2, 4, and 6 weeks after birth (Supplemental Figure 10). Raw and processed microarray data are available through the NCBI's Gene Expression Omnibus (GEO) database (GEO GSE57108). These analyses revealed 
A

C57BL/6-Rag1-/

$+/+$

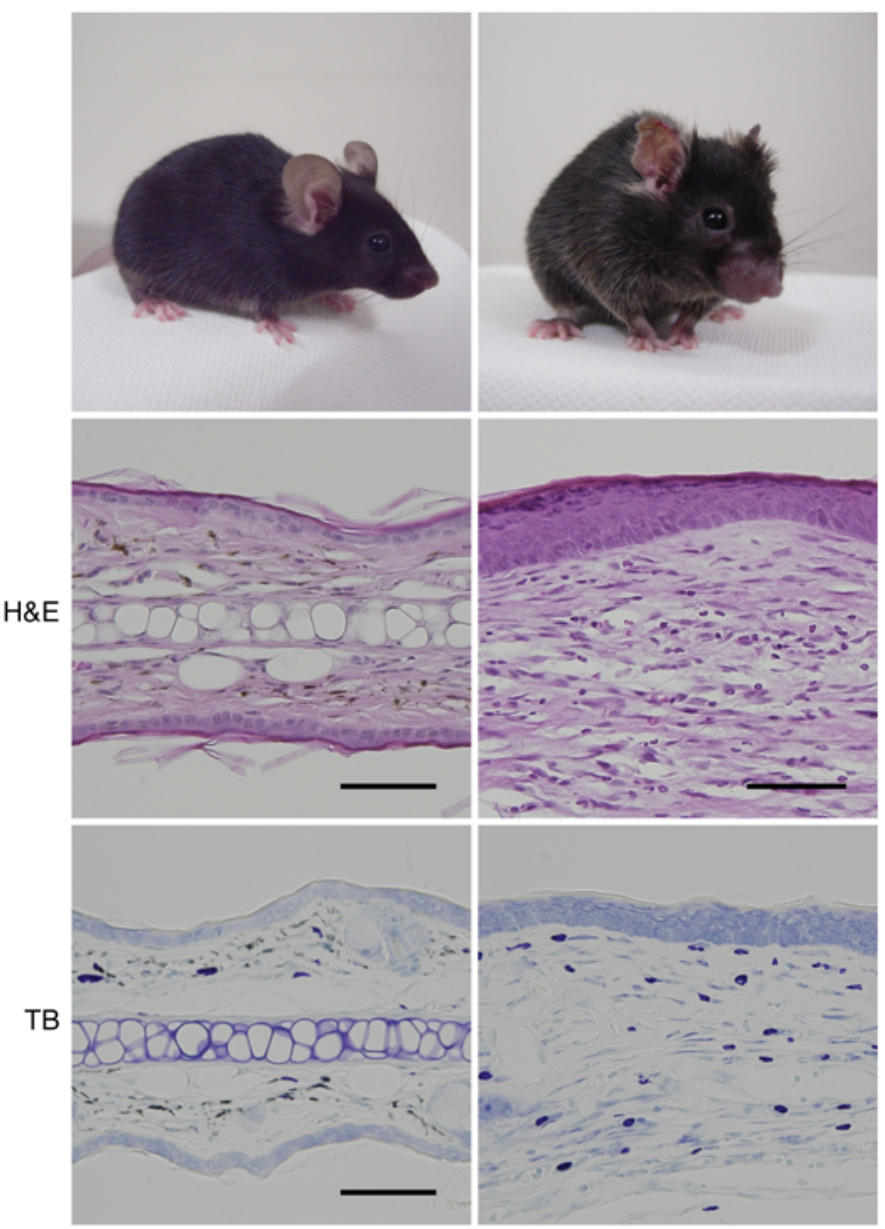

B

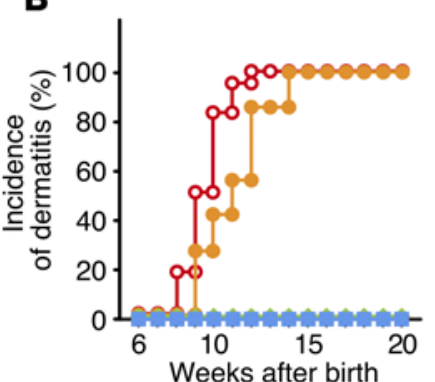

C57BL/6-Rag 1-1-

Jak $1^{\text {Spade/Spade }}$

C

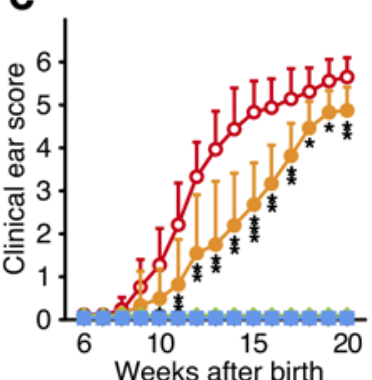

D
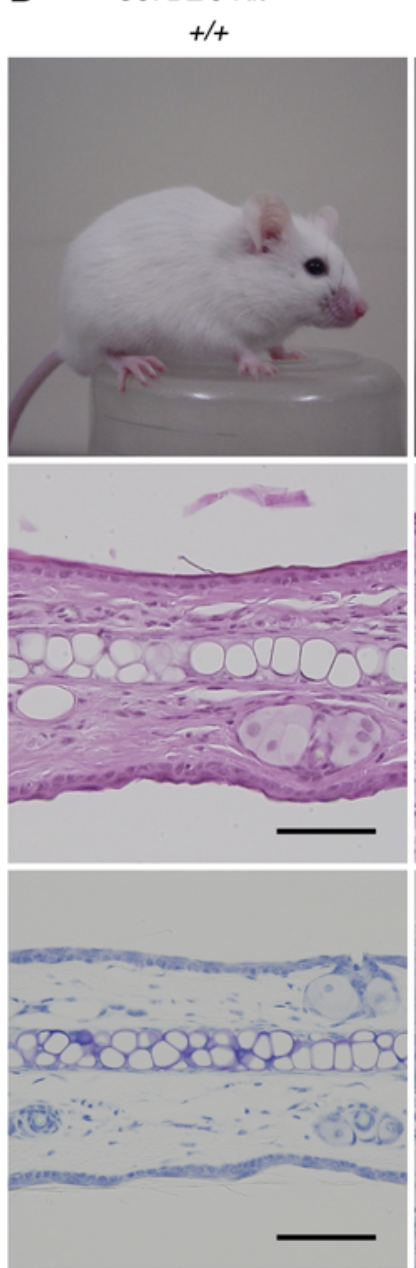

E

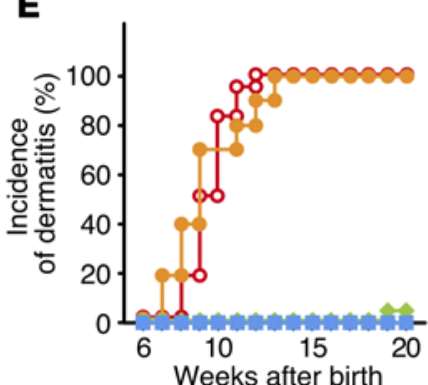

$\mathbf{F}$

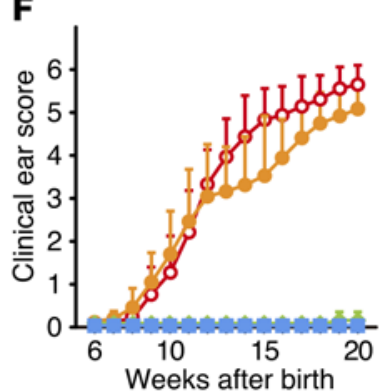

-o- Kitt// Jak $1^{\text {Spade/spade }}(n=12)$

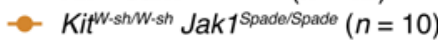

- Kitt.shw-sh Jak $1^{+/ \text {Spade }}(n=18)$

$=K i t^{W \cdot s h W \cdot s h} \operatorname{Jak}^{+/+}(n=10)$

Figure 4. Dermatitis development in $\mathbf{T}$ and $\mathbf{B}$ cell- or mast cell-deficient Jak $1^{\text {5pade/spade }}$ mice. (A and $\left.\mathbf{D}\right)$ Physical and histological appearance of dermatitis in Jak $7^{\text {Spade/Spade }}$ mice on a Rag1-/- (A) or Kit ${ }^{\text {W-sh/W-sh }}$ (D) background. Upper panels show photographs of the indicated mice. Lower panels show photomicrographs of H\&E- or TB-stained ear skin histological sections from the indicated mice. Scale bars: $100 \mu \mathrm{m}$. (B, C, E, and F) Incidence (B and

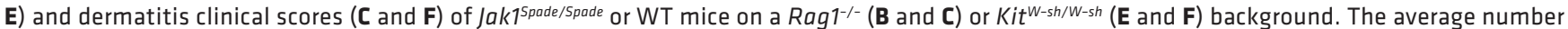
of weeks to onset was $11.14 \pm 1.77$ for Rag1/- Jak $1^{\text {Spade/Spade }}$ mice and $9.67 \pm 0.98$ for Jak $1^{\text {Spade/Spade }}$ mice $(P=0.031$, by 2 -tailed Student's $t$ test $)$, and 9.55 \pm 2.11 for Kit ${ }^{W-s h / W-s h} J a k 1^{\text {Spade/Spade }}$ mice and $9.67 \pm 0.98$ for Jak $1^{\text {Spade/Spade }}$ mice $(P=0.860$, by 2-tailed Student's $t$ test $) .{ }^{*} P<0.05,{ }^{* *} P<0.01$, and ${ }^{* * *} P$ $<0.001$, relative to the Jak $1^{\text {spade/spade }}$ single-mutant mice in C, by Mann-Whitney $U$ test. 
that expression of a group of genes, including keratinocyte differentiation-specific molecules, macrophage activationspecific molecules, and mast cell-specific molecules, was upregulated in the skin tissue of mutant mice (Supplemental Table 1). Among this group of genes, we focused on the expression levels of serine proteases, because it has been reported that a defect in a skin serine protease-specific inhibitor caused hyperactivation of serine proteases, which in turn resulted in an atopic dermatitis-like disease in Netherton syndrome (21). The expression level of several serine proteases was dramatically upregulated in the weeks leading up to dermatitis onset in Jak1 $1^{\text {Spade/spade }}$ mice (Figure 7A). Validation of these results by quantitative reverse transcription PCR (qRT-PCR) revealed that $\operatorname{Klk6}(22,23)$ and marapsin (also known as Prss27) (24) mRNAs were expressed at 10 -fold higher levels in the skin from Jak1 $1^{\text {Spade/spade }}$ mice than in skin from WT mice (Figure 7, B and C). The 5' upstream regions of the Klk6 and marapsin genes contain a binding consensus sequence for STAT family transcription factors, therefore, we next investigated whether the JAK1 ${ }^{\text {spade }}$ mutation affects the transcriptional activity of these protease genes. To this end, we cotransfected 293T cells with a WT or Jak1 ${ }^{\text {Spade }}$ cDNA and a luciferase reporter construct containing the promoter regions of Klk6 or marapsin. Luciferase activity was significantly increased in transfectants expressing the Jak1 $1^{\text {spade }}$ gene compared with those expressing WT Jak1 (Figure 7, D and E). This result suggests that the hyperactivity of the JAK $1^{\text {spade }}$ mutant directly affects the transcriptional regulation of protease genes and may induce overexpression of these proteases in the skin.

To confirm the relationship between JAK1 hyperactivation and protease overexpression, we painted the ear skin of Jak1 $1^{\text {spade/Spade }}$ mice with a JAK inhibitor or with solvent alone every 2 days from 4 weeks of age. This treatment considerably delayed the onset of disease and attenuated the disease progression rate (Figure $7 \mathrm{~F}$ ), such that approximately $50 \%$ of the mutants had no dermatitis symptoms at 12 weeks of age (Figure $7 G$ ). This therapeutic benefit coincided with decreased expression of pY-JAK1 and Ki67 compared with expression levels detected in mutant mice treated with solvent alone (Figure $7 \mathrm{H})$. Taken together, our results indicate that the development of dermatitis in Jak1 $1^{\text {spade/spade }}$ mice is mediated by JAK1 hyperactivity in epidermal keratinocytes. We examined the expression levels of Klk6 and marapsin mRNA in mouse ear skin by qRT-PCR and found that they were lower in inhibitor-painted ears than in solvent-painted ears (Supplemental Figure 11, A and $\mathrm{B})$. In contrast, the expression level of these proteases in the petrolatum-painted ears of mutant mice was comparable to that of nontreated Spade homozygotes (Supplemental Figure 11, $\mathrm{C}$ and $\mathrm{D})$. These results indicate that the decreased expression of proteases observed in JAK inhibitor-painted skin was not the result of delayed disease onset but rather was due to inhibition of JAK1 activation.

Hyperphosphorylation of JAK1 in the skin of patients with atopic dermatitis. The above results suggested that JAK1 hyperphosphorylation might also be seen in the epidermis of patients with dermatitis. We examined 6 independent atopic dermatitis skin biopsies from patients and detected increased epidermal JAK1 phosphorylation in 4 of the biopsies (Figure 8).

\section{Discussion}

In this study, we established a new dermatitis mouse model induced by a point mutation $(\mathrm{R} 878 \mathrm{H})$, designated the Spade mutant, in the Jak1 tyrosine kinase near the ATP-binding motif of the tyrosine kinase domain. Although the reported Jak1deficient mouse has no phenotype in skin development (25), the Spade point mutation constitutively hyperactivates the JAK1/ STAT signal transduction pathway in the epidermis, which in turn results in aberrant differentiation and hyperproliferation of keratinocytes, resulting in a skin barrier defect. We observed that JAK1 hyperactivation in the epidermis is accompanied by overexpression of serine proteases, including members of the kallikrein-related peptidases (KLK) family. KLK family serine proteases participate in corneodesmosome degradation during skin turnover (26), and their overexpression or deletion of their physiologic inhibitors induces a dermatitis phenotype in humans (27) and mice (28). In addition, we observed that painting of a JAK inhibitor onto Spade homozygous mutant ears decreased the expression of these proteases and delayed the onset of dermatitis. Our results provide evidence for the overexpression of such serine proteases by activation of STATs via a signaling cascade through an activated JAK1 pathway, defining a linkage between JAK1 and skin barrier homeostasis via serine protease activation. It is interesting to note that, recently, several JAK inhibitors have been administrated to patients with psoriasis and are showing beneficial results for the treatment of psoriasis skin inflammation (29). In these cases, JAK inhibitors are thought to be acting via immune cell function regulation (30) rather than affecting skin tissue, however, our study suggests that the epidermal keratinocyte proliferation or differentiation may also be a target of those reagents. Since hyperphosphorylation of epidermal JAK1 was detected in a subset of of skin samples from patients with atopic dermatitis, it will be interesting to determine whether JAK inhibitors might also prevent the onset of human atopic dermatitis.

In addition to the direct effect on skin, we have found that BM cells with JAK1 hyperactivation also affect the onset of dermatitis in Jak1 ${ }^{\text {Spade/Spade }}$ mice. Since proliferation and differentiation of the skin epidermis is affected by various cytokine signals, intrinsic or from other cells $(7,31-33)$, it seems plausible that distinct cytokines activating the JAK1 signal transduction system in BM-derived cells may play some role in skin barrier maintenance. In fact, there is a report of elevated KLK6 and marapsin expression levels in reorganized human keratinocytes upon in vitro stimulation with IL-19, IL-20, IL-24, and IL-22, cytokines produced by dermal macrophages, DCs, and Th17 cells (34). As they also participate in epidermal keratinocyte proliferation and psoriasis development (35), these cytokines are plausible candidates for regulators of skin barrier maintenance via JAK1 signal transduction in both normal and disease states.

It is thought that the acquired immune system modulates the onset and progression of atopic dermatitis, in particular via Th2 immune responses driven by $\mathrm{T}$ and $\mathrm{B}$ lymphocytes or mast cells $(31,36,37)$. However, dermatitis developed in Spade-mutant mice lacking these immune cells, supporting the idea that initiation of the disease is independent of the activity of lymphocytes or mast cells. Nonetheless, WT BM cells transferred into Spade-mutant 

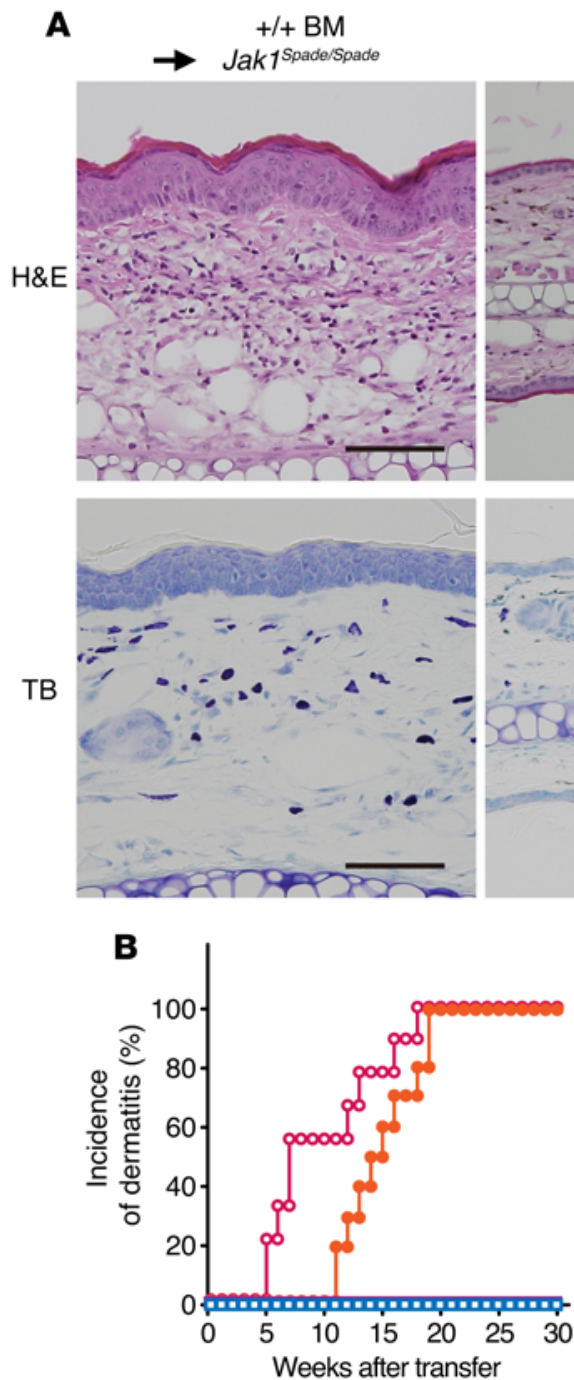
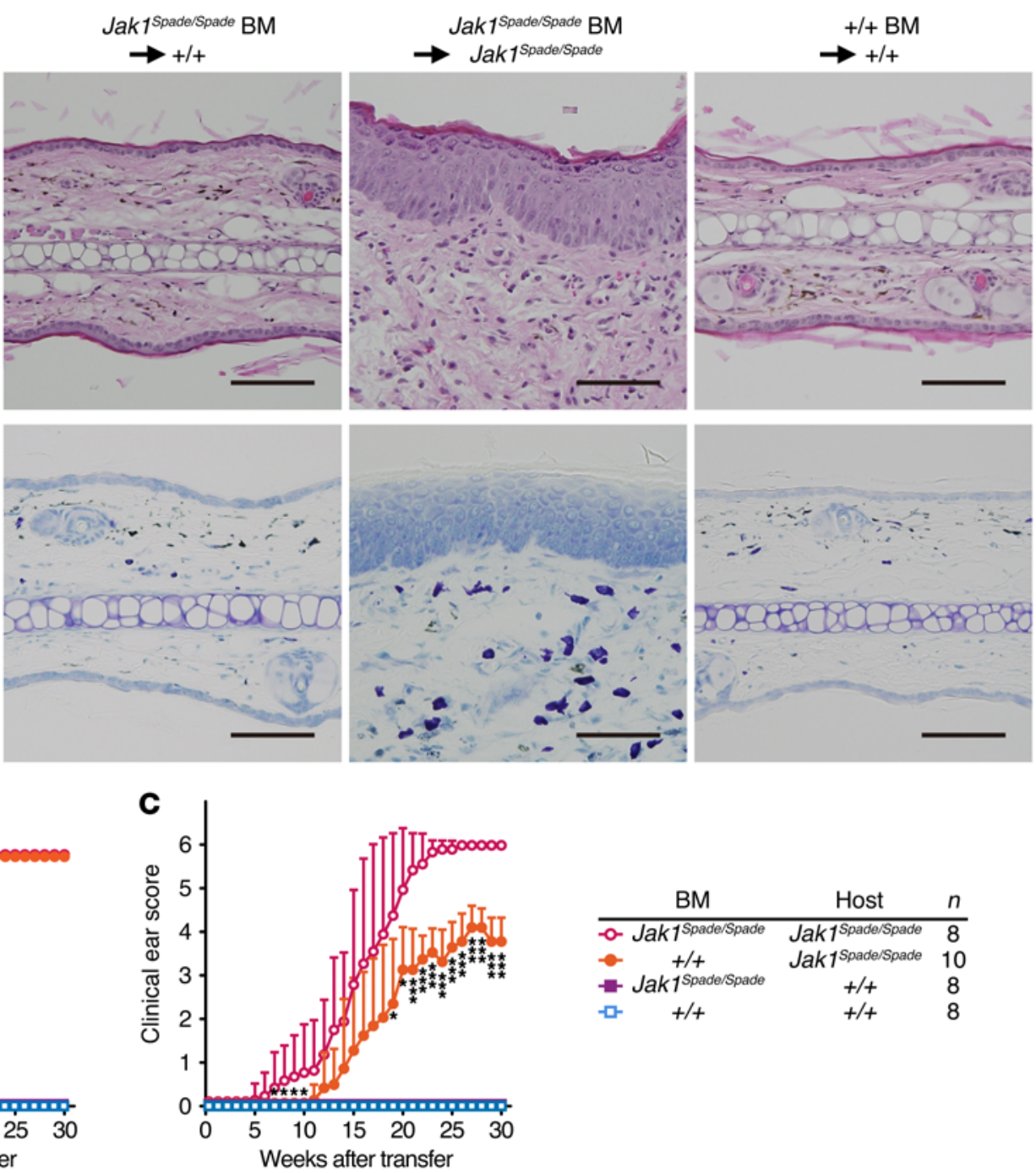

Figure 5. Dermatitis development in BM-transferred mice. (A) Photomicrographs showing H\&E and TB staining of ear skin histological sections from BM chimeric mice in various combinations. Scale bars: $100 \mu \mathrm{m}$. (B and C) Longitudinal analyses of dermatitis incidence (B) and clinical scores (C) after BM transplantation between Jak $1^{\text {Spade/Spade }}$ and WT mice. ${ }^{*} P<0.05$ and ${ }^{* * *} P<0.001$, relative to Jak $1^{\text {Spade/Spade }} \mathrm{BM} \rightarrow$ Jak $1^{\text {Spade/Spade }}$ chimeric mice, by Mann-Whitney $U$ test.

mice delayed the onset of disease, suggesting the possible importance of some components of the innate immune response in the initiation of dermatitis (38).

In addition to genetic factors, we have found that an environmental factor, skin barrier functional repair by petrolatum painting, prevented the onset of dermatitis. Under these conditions, infiltration of neutrophils and monocytes to the dermis of the ear was blocked, in keeping with delayed dermatitis onset, suggesting that the infiltration of myeloid cells might play an important role in the onset of dermatitis. On the other hand, permeability barrier disruption induced experimentally by tape stripping did not accelerate dermatitis development in homozygous or heterozygous mice. These results indicate that dermatitis is initially induced by the genetic defect, which disrupts skin barrier homeostasis, but that environmental factors that induce innate immune cell activation also strongly modulate disease onset and progression. Recently, several studies have suggested that the development of human juvenile atopic dermatitis could be prevented by emollient treatment $(39,40)$. From this point of view, our Spade-mutant mice should be a useful model to investigate the presymptomatic pathological changes in the skin under emollient treatment, which is difficult to investigate in humans.

While the present article was in preparation, Sabrauski et al. reported that a missense point mutation (S645P) in the pseudokinase domain of the Jak1 gene resulted in dermatitis accompanied by STAT3 activation (41). However, there are important differences between our results and theirs with respect to disease onset and phenotype. Their Jak $1^{1645 P}$ mutant did not develop spontaneous dermatitis until approximately 4 months of age, which was later than the disease onset observed in our Jak1 19ade (R878H) mutant mice. Moreover, the S645P mutation results in a systemic lupus erythematosus-like (SLE-like) disease, coincident with the elevation of serum Ig isotypes and production of autoantibodies. As signal transduction by JAK1 involves multiple downstream signaling pathways (42), it is conceivable that the 2 JAK1 mutations, S645P and R878H, promote JAK1 gain of function, but with variably enhanced activation of different downstream signaling pathways. In fact, it has been reported that point mutations such as 
A

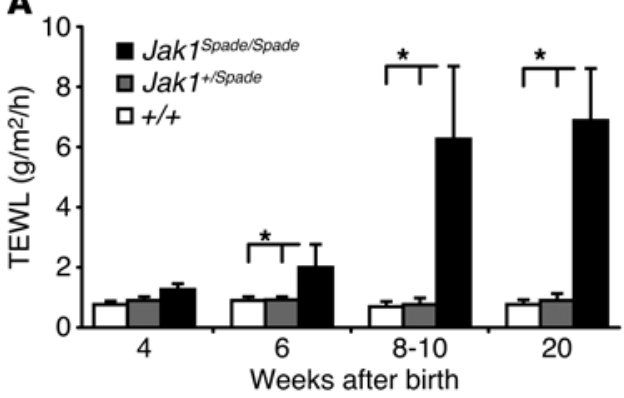

B
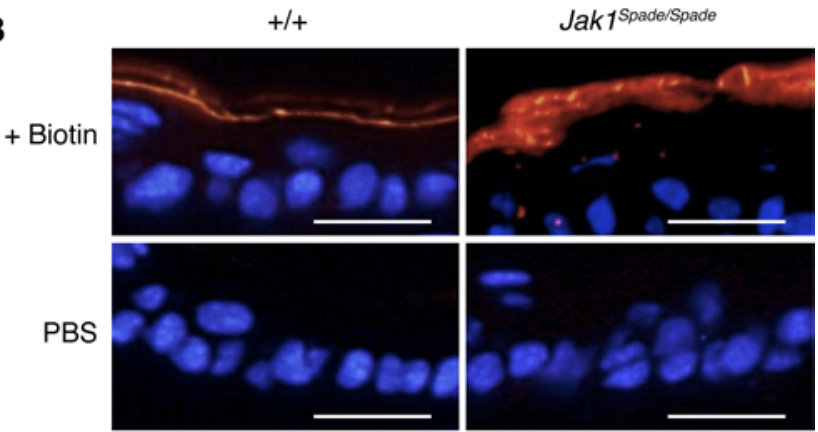

C

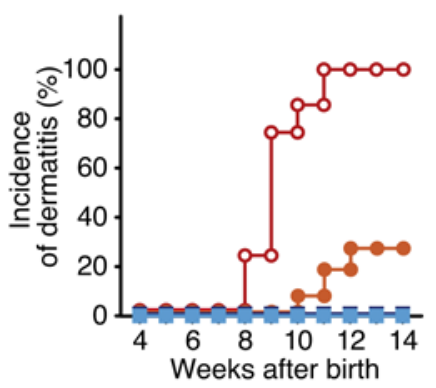

D

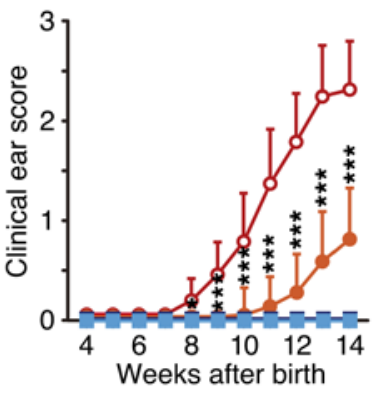

Application

None

Petrolatum

None

Petrolatum
E

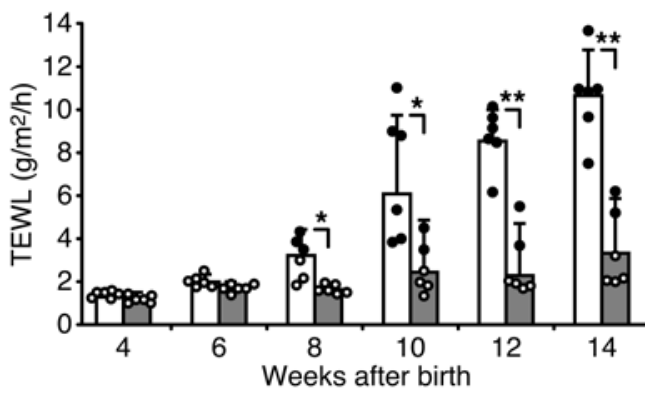

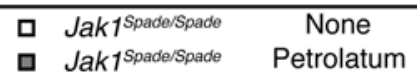

- Nondiseased mouse

- Diseased mouse

Figure 6. Dermatitis in Spade-mutant mice is due to a defect in skin barrier function that coincides with abnormal expression of serine proteases in mutant skin. (A) Longitudinal analysis of the TEWL rate in the ear skin of Jak1 $1^{\text {spade/spade }}$, Jak $1^{+/ \text {spade }}$, and WT mice. Error bars represent the mean \pm SD of 6 mice per group. ${ }^{*} P<0.05$, by 1-way ANOVA, followed by Bonferroni's test. (B) Fluorescence micrographs showing the infiltration of biotin particles into the skin of 6-week-old Jak15pade/Spade and WT mice. Scale bars: $20 \mu \mathrm{m}$. (C and D) Longitudinal analyses of dermatitis incidence (C) and clinical scores (D) in mice treated from 4 weeks to 14 weeks of age with a topical application of petrolatum 3 times per week. Data represent the mean \pm SD of 8 mice per group. ${ }^{*} P<0.05$ and ${ }^{* * *} P<0.001$, relative to nontreated Jak1 ${ }^{\text {spade/spade }}$ mice, by Mann-Whitney $U$ test. (E) Longitudinal analysis of TEWL in Jak15pade/spade mice treated with a topical application of petrolatum or left untreated as a control. Each circle represents the TEWL level of an individual mouse, and white circles and black circles represent nondiseased and diseased mice, respectively. Error bars represent the mean \pm SD of 6 mice per group. ${ }^{*} P<0.05$ and ${ }^{*} P<0.01$, by 2 -tailed Student's $t$ test.

A634D in the pseudokinase domain (JH2) of human JAK1 and the tyrosine kinase domain mutation R879C (corresponding to mouse $\mathrm{R} 878 \mathrm{H})$ displayed different cellular phenotypes in transfection studies $(43,44)$. Further analysis is needed to clarify how the quality of signal transduction via JAK1 is different between pseudokinase and kinase domain mutations.

We have investigated whether the equivalent Jak1 $1^{\text {spade }}(\mathrm{R} 878 \mathrm{H})$ mutation has been reported in human JAK1; however, there have been no reports showing such a gain-of-function mutation in the coding region of the JAK1 gene as a germline mutation (45). Instead, there was a case report describing a somatic mutation of $J A K 1$, resulting in an arginine-to-histidine change at aa position $879, J A K 1^{R 879 H}$, which corresponds to the same point mutation as that found in the Spade mutant (R878H), in adult pre-T cell-type acute lymphoblastic leukemia (T-ALL) $(43,45)$. It was reported that approximately $18 \%$ of adult patients with early-onset T-ALL have a mutation in the JAK1 coding region and that all of the mutants examined in vitro showed a gain-of-function phenotype (43). In this context, the proliferation of Spade thymocytes was elevated; however, we did not detect any tumor development in more than 100 homozygous mice that were observed up until 76 weeks of age. This may reflect multiple incidental alterations in the genome in early T-ALL genesis (46), and JAK1 ${ }^{R 878 H}$ may not be a driver mutation.

In future studies, careful analysis of dermatitis development and immune responses, not only in Spade mice but also in Jak $1^{5645 P}$ mice, will reveal the signaling pathways critical for skin barrier homeostasis and disease development, findings that will be a helpful tool for human skin inflammatory disease investigations.

\section{Methods}

Mice. The detailed ENU mutagenesis protocol has been previously described (13). C57BL/6J and $\mathrm{C} 3 \mathrm{H} / \mathrm{HeJ}$ mice were purchased from CLEA Japan. C57BL/6-Kit ${ }^{W-s h / W-s h}$ mice were provided by the RIKEN BioResource Center (deposited by Kesuke Oboki, National Research Institute for Child Health and Development, Tokyo, Japan). C57BL/6-Rag1/-- mice were bred in our facility. All mice were maintained under SPF conditions 


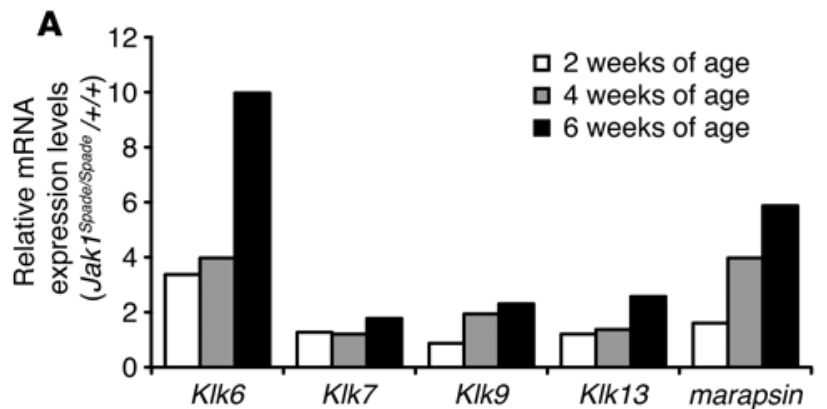

D

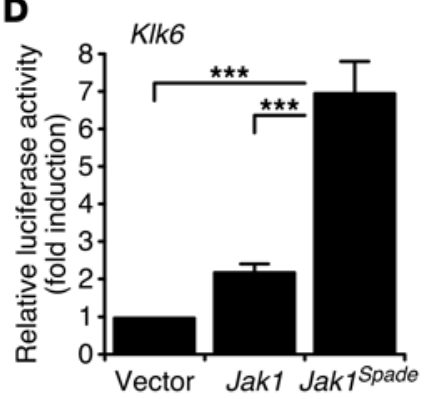

E

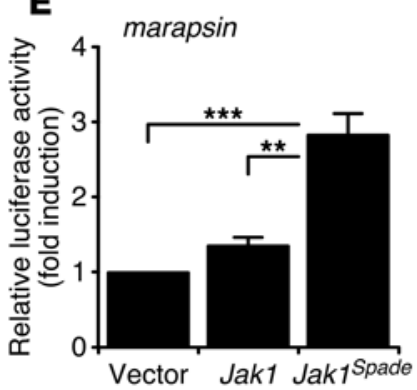

H
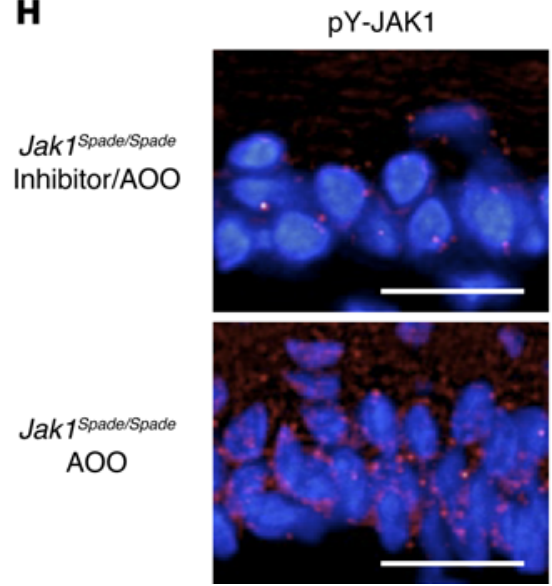

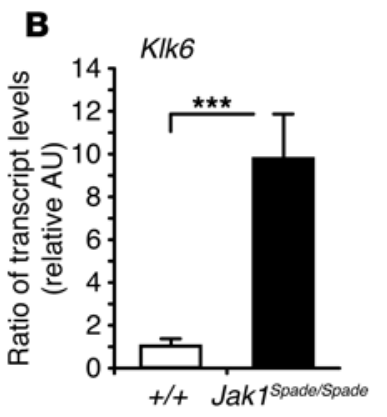

$\mathbf{F}$

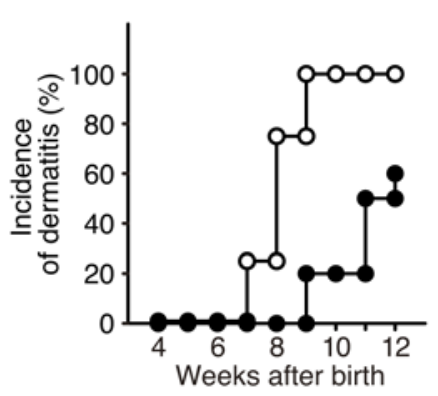

G

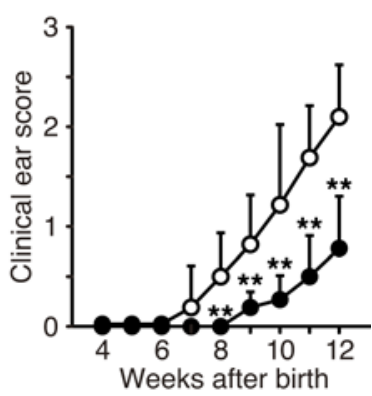

K6
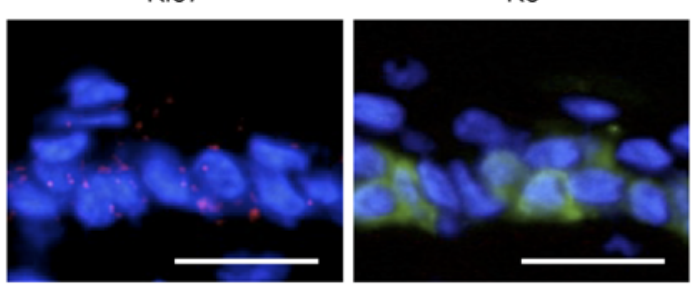

\begin{tabular}{|c|c|}
\hline Genotype & Application \\
\hline - J Jak1 $1^{\text {Spade/Spade }}$ & $\mathrm{AOO}$ \\
\hline
\end{tabular}
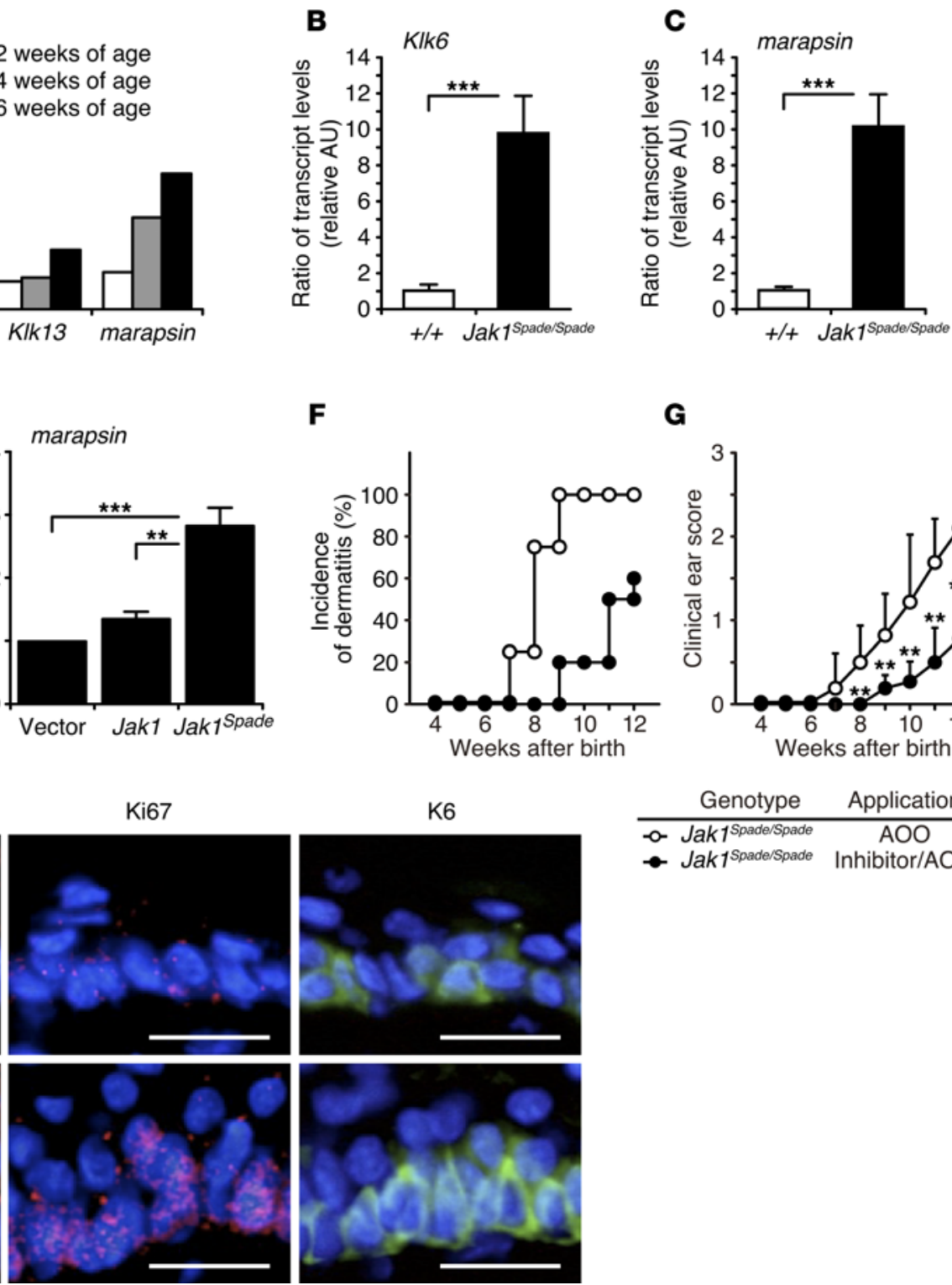

Figure 7. Overexpression of serine proteases in Spade-mutant skin and their role in dermatitis development. (A) Changes in mRNA levels of serine proteases determined by microarray analysis. Each bar depicts the ratio of mRNA expression level in the ear skin of Jak $7^{\text {Spade/spade }}$ mice to that in WT mice

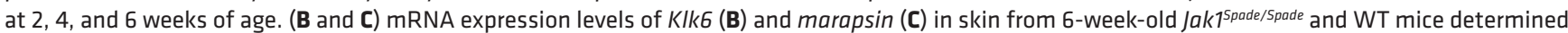
by qRT-PCR. Error bars represent the mean \pm SD of 6 mice per group. ${ }^{* *} P<0.001$, by 2 -tailed Student's $t$ test. (D and E) Luciferase assay for KIk6 (D) and marapsin (E) promoter activity in 293 T cells expressing Jak $1^{\text {spade }}$ or WT Jak1 cDNA. (F and $\left.\mathbf{G}\right)$ Topical application of the JAK inhibitor was done on mice 3 times per week, from 4 to 12 weeks of age. Dermatitis incidence $(\mathbf{F})$ and clinical ear score (C) for Jak $7^{\text {spade/Spade }}$ mice treated with the JAK inhibitor in AOO or solvent alone. Each circle represents the mean \pm SD of 8 mice per group. ${ }^{*} P<0.01$, by Mann-Whitney $U$ test. The average number of weeks to onset was $10.71 \pm 1.25$ for mice treated with inhibitor and $8.00 \pm 0.76$ for mice treated with solvent alone $(P=0.00018$, by 2-tailed Student's $t$ test). (H) Frozen ear sections from WT and Jak $7^{\text {Spade/spade }}$ mice 3 weeks after JAK inhibitor or solvent treatment were stained with anti-pY-JAK1 and Ki67 (both red) and K6 (green), and nuclei were counterstained with DAPI (blue). Scale bars: $20 \mu \mathrm{m}$.

Clinical scoring of dermatitis. A clinical severity score was determined as the sum of the individual scores for scratching behavior and the symptoms in 4 regions (right and left ears, face excluding ears, and other regions) (12). Scratching behavior was graded as 0 (rarely); 1 (sometimes; <30 seconds of scratching during a 120second observation); 2 (frequently; 30-90 seconds); or 3 (constantly; $>90$ seconds). Symptoms were graded as 0 (none); 1 (mild; skin dryness, erythema, hemorrhage, scaling, and excoriation); 2 (moderate; erosion, crust, and fissures/rhagades on the ear); or 3 (severe; thickening/lichenification and deformed/defective ears). Disease onset was defined as a clinical score above 1 .

Mapping and sequencing. Phenodeviants that showed skin anomalies were crossed with $\mathrm{C} 3 \mathrm{H} / \mathrm{HeJ}$ mice to test for phenotype transmission and for genetic mapping using SNPs (9). For sequencing, we focused on Jak1 among the candidate genes identified by the PosMed bioinformatics-based search engine (47). Genomic DNA was amplified by PCR and sequenced using Jak1-specific primers (Supplemental Table 2). 


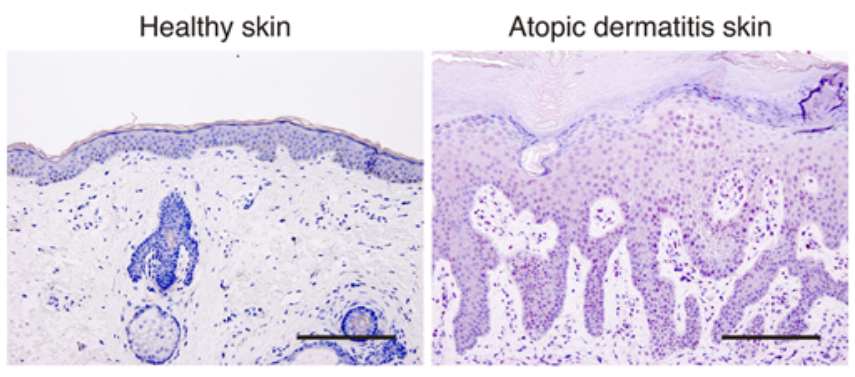

Figure 8. Immunohistological analysis of JAK1 activation in the skin of a patient with atopic dermatitis. Photomicrographs of skin histological sections from a patient with atopic dermatitis and a healthy control immunostained for PY-JAK1. Hyperphosphorylation of JAK1 was detected in 4 of 6 samples from patients with atopic dermatitis. A representative photograph is shown. Scale bars: $100 \mu \mathrm{m}$.

Generation of BM chimeric mice. Recipient mice were lethally irradiated at $9.5 \mathrm{~Gy}$ with a ${ }^{137} \mathrm{Cs}$ source and injected i.v. with $1.0 \times$ $10^{7} \mathrm{BM}$ cells from the the Jak $1^{\text {Spade/Spade }}$ or WT donor mice 8-10 hours after irradiation. The recipient mice were given drinking water containing $0.5 \mathrm{mg} / \mathrm{ml}$ neomycin sulfate and $0.3 \mathrm{mg} / \mathrm{ml}$ tetracycline hydrochloride (Calbiochem).

Generation of Jak1-mutant mice. A genomic clone RP23-25A20 was obtained from a C57BL/6J mouse bacterial artificial chromosome (BAC) clone collection (Invitrogen). The 2.4-kbp BxtXI-Aor51HI and 4.3-kbp Aor51HI-EcoRV DNA fragments containing exons 16-17 and 18-22, respectively, of the Jak1 gene were subcloned into the pGEM-T Easy Vector (Promega). We introduced the mutation (R878H, from CGT to CAT) in exon 19 of Jak1 using a recombinant PCR technique. The targeting vector was constructed using the pMC1neoPolyA (Stratagene) plasmid. A loxP-flanked Neo cassette was cloned into the Aor51HI site of intron 17 of Jak1. The thymidine kinase gene was inserted upstream of the $5^{\prime}$ arm. M1 ES cells were transfected with the linearized vector by electroporation. The resultant G418- and ganciclovir-resistant clones were screened using PCR and Southern blot analysis with two ${ }^{32} \mathrm{P}$-dCTP-labeled DNA probes. The following primer sets were used for PCR screening of embryonic stem (ES) cells: for 5'-side PCR amplification, 5'-GAGTGGAGAAAGCTGACCTGTG-3' and 5'-CGCCTTCTATCGCCTTCTTGAC-3'; for 3'-side PCR amplification, 5'-CAGTCATAGCCGAATAGCCTC-3' and 5'-AGCAGTCCAGAGGCTCACAGCAAC-3'. Southern blot analyses of candidate ES cells were performed using 2 probes taken from genomic DNA containing the Jak1 gene (for the $5^{\prime}$ probe of $810 \mathrm{bp}$, 5'-CTAGCATGATGAGACAGGTT-3' and 5'-TCCGGTGCATGAAGAGATCC-3'; for the $3^{\prime}$ probe of $490 \mathrm{bp}$, 5'-CAGCATGAATGGCCAGAGTCC-3' and 5'-ATTGGTCCATAACAGCACATC-3'). The genomic mutation $(\mathrm{R} 878 \mathrm{H})$ was confirmed by sequencing. Germline chimeric mice were generated by aggregation methods, and mice with a high ES cell chimera contribution were crossed with C57BL/6J mice to produce heterozygous mutants. The heterozygous mutants were mated with mice expressing the Cre recombinase protein to remove the loxP-flanked Neo cassette.

Western blotting. MEFs were stimulated with either IL-6 (50 ng/ $\mathrm{ml})$ or IFN- $\alpha(500 \mathrm{U} / \mathrm{ml})$. The cell lysates were incubated with JAK1 (BD Biosciences; catalog 610231, clone 73/JAK1) mAb and STAT3 (Cell Signaling Technology; catalog 9132) or STAT1 (Cell Signaling
Technology; catalog 9172) polyclonal Abs, together with protein A sepharose (GE Healthcare). The immunoprecipitates were separated by gradient SDS-PAGE, followed by transfer onto a PVDF Immobilon P Membrane (EMD Millipore). The membranes were incubated with pTyr (Upstate; catalog 05-777, clone 4G10); JAK1 (BD Biosciences; catalog 610231, clone 73/JAK1); pY-STAT3 (Cell Signaling Technology; catalog 9131); STAT3 (Cell Signaling Technology; catalog 9132); pY-STAT1 (Cell Signaling Technology; catalog 9171); or STAT1 (Cell Signaling Technology; catalog 9172) Abs. After washing, the membranes were incubated with HRP-conjugated goat anti-rabbit IgG (Zymed; catalog 65-6120). The immune complexes were visualized using a chemiluminescence system (PerkinElmer Life Sciences). The density of each band was quantified using ImageJ software (NIH).

In vitro kinase assay. JAK1 kinase activity was analyzed as previously described (14). Briefly, 293T cells expressing a chimeric receptor composed of the extracellular domain of the granulocyte-CSF (G-CSF) receptor and the intracellular domain of gp130 were transfected with the R878H mutant or WT JAK1, followed by stimulation with G-CSF (100 ng/ml). After stimulation, HA-tagged JAK1 was immunoprecipitated, washed, and incubated with kinase buffer and recombinant STAT3 protein (Active Motif) as a substrate. Phosphorylation was analyzed by immunoblotting using pY-JAK1 (Cell Signaling Technology; catalog 3331); JAK1 (BD Biosciences; catalog 610231, clone 73/JAK1); pY-STAT3 (Cell Signaling Technology; catalog 9131); and STAT3 (Cell Signaling Technology; catalog 9132) Abs.

Barrier function assays. TEWL was evaluated on the ear using the Tewameter TM 300 electrolytic water analyzer (Courage + Khazaka electronic $\mathrm{GmBH}$ ). For permeability assays, an isotonic solution containing NHS-LC-biotin (Pierce) in PBS was applied on the ear. After a 60-minute incubation, the ear skin was dissected and frozen. Frozen sections were stained with Alexa Fluor 546-conjugated streptavidin (Invitrogen) and counterstained with DAPI (Vector Laboratories).

Topical application of a JAK inhibitor and skin-protective agent. JAK Inhibitor I ( $150 \mu \mathrm{M}$; Calbiochem) in 10- $\mu$ l aliquots of acetone and olive oil (AOO) (4:1) or petrolatum was topically applied 3 times per week to the ventral and dorsal portions of the ear for the duration of the treatment.

Histological and immunohistological analysis. Mouse ear skin was fixed with $4 \%$ paraformaldehyde, embedded in polyester wax (VWR International), and sectioned for $\mathrm{H} \& \mathrm{E}$ or toluidine blue (TB) staining. For immunohistology, frozen ear specimens were sectioned and fixed by microwave irradiation. After blockade with $5 \%$ normal mouse serum in 3\% BSA in PBS, slides were stained with pY-JAK1 (Cell Signaling Technology; catalog 3331); Ki67 (Abcam; catalog ab15580); or K6 (Covance; catalog PRB-169P) Abs, followed by incubation with FITC-conjugated swine anti-rabbit Igs (Dako; catalog F0205) or Cy3-conjugated goat anti-rabbit IgG (Zymed; catalog 81-6115) secondary Abs. Slides were counterstained with DAPI (Vector Laboratories).

Immunohistological analysis of human skin. Formalin-fixed, paraffin-embedded skin tissue sections from patients with atopic dermatitis or from healthy controls were antigen retrieved with $10 \mathrm{mM}$ sodium citrate buffer ( $\mathrm{pH}$ 6.0) and then probed with pY-JAK1 Ab (Sigma-Aldrich; catalog SAB4504446). Sections were incubated with biotinylated secondary anti-rabbit IgG Ab, followed by an avidinbiotinylated alkaline phosphatase (AP) complex (VECTASTAIN ABC-AP Rabbit IgG Kit; Vector Laboratories; catalog AK-5001). The $\mathrm{Ab}$ was detected with red AP substrate, and nuclei were counterstained with hematoxylin. 
ELISA. Serum Ig and histamine levels were determined using an ELISA kit (Bethyl and MBL).

Real-time reverse transcription $q R T-P C R$ analysis. Ear tissue was disrupted using a bead mill (Tomy Seiko; MS-100R), and total cellular mRNA was isolated using an RNeasy Fibrous Tissue Kit (QIAGEN) according to the manufacturer's instructions. RNA was reverse transcribed using the SuperScript VILO cDNA Synthesis Kit (Invitrogen). For the quantitative analysis of gene expression, qRT-PCR was performed on an ABI 7300 Real-Time PCR System with TaqMan Gene Expression Assays (KLK6; Mm00478322_m1, PRSS27; Mm00841353_g1) and TaqMan Gene Expression Master Mix (both from Applied Biosystems). Samples were normalized to the mouse ribosomal housekeeping gene RPL19 (Mm02601633_g1) and reported according to the $2^{-\Delta \Delta \mathrm{Ct}}$ method, where $\Delta \mathrm{Ct}=\mathrm{Ct}$ of the target gene $-\mathrm{Ct}$ of RPL19, and $\Delta \Delta \mathrm{CT}=$ $\Delta \mathrm{CT}$ of the sample $-\Delta \mathrm{CT}$ of the WT sample.

Total RNA was extracted from MEFs using Sepasol-RNA I (Nacalai Tesque) and reverse transcribed with oligo-(dT) primers and reverse transcriptase (ReverTra Ace; Toyobo). SYBR Green PCR Master Mix (Applied Biosystems) was used for qRTPCR analysis. Transcript levels were normalized to those of the gene encoding Gapdh. Primer sequences were as follows: Socs1, 5'-CACCTTCTTGGTGCGCG-3' and 5'-AAGCCATCTTCACGCTGAGC-3'; Socs3, 5'-CCCAAGGCCGGAGATTTC-3' and 5'-GGAGCCAGCGTGGATCTG-3'; and Gapdh, 5'-AGCTGAACGGGAAGCTCACT-3', and 5'-TGAAGTCGCAGGAGACAACC-3'.

Dual-Luciferase reporter assay. Genomic DNA fragments of the Klk6 (-1859 to -1$)$ and Prss27 (-1012 to -1$)$ promoter regions containing a STAT3-binding site (TTCCCRKAA) (48) were amplified from genomic DNA by PCR using primers with an appropriate restriction site (Klk6, 5'-CTTGCGAGATACAAATAACA- $3^{\prime}$ and 5'-CTTGAGAGGGTAAGCCAACG-3'; and Prss27, 5'-GCTGCACCCTCTGCTCCTGCGGG-3', and 5 '-GTTCTCTGGCTTGGTGGACG-3'). The amplified products were subcloned into the pGL3 basic vector (Promega) containing a firefly luciferase reporter gene. The authenticity of the clones was confirmed by DNA sequencing. 293T cells stably transfected with R878Hmutant JAK1 (pCMV-mutant JAK1), WT JAK1 (pCMV-JAK1), or pCMV vector alone were cotransfected with a STAT3 expression vector (pEFBOS-STAT3), a Renilla luciferase expression vector (pRL-TK; Promega), and either pGL3-KLK6 or pGL-PRSS27 using Lipofectamine 2000 (Invit- rogen). Twelve hours after transfection, cell extracts were prepared, and reporter gene assays were conducted with the Dual-Luciferase Reporter Assay System (Promega) according to the manufacturer's instructions. Firefly luciferase activity was normalized to Renilla luciferase values.

Additional experimental procedures are described in the Supplemental Methods.

Statistics. Statistical significance was calculated by a 2-tailed Student's $t$ test or a Mann-Whitney $U$ test or by 1-way ANOVA, followed by Bonferroni's test for multiple comparisons. $P$ values of 0.05 or less were considered significant.

Study approval. All animal experiments were carried out under the guidance of the Regulations for the Animal Experiments (RIKEN Yokohama Institute, Yokohama, Japan) and were approved by the Animal Experiment Committee of RIKEN (Yokohama Institute, Yokohama, Japan).

After informed consent was provided, human skin samples were obtained from patients with atopic dermatitis or from unused portions of grafts, most of which had been intended for use in surgery. The protocol was approved by the Ethics Committee for Human Studies of Kyoto University Graduate School of Medicine, which stipulates adherence to the rules laid out in the Declaration of Helsinki.

\section{Author contributions}

TY, TF, KN, MN, MM, IM, TD, SF, KK, MK, HO, TH, OO, HK, SW, and HY designed and/or performed experiments. TY, SN, and BHB analyzed data. TY and HY wrote the manuscript.

\section{Acknowledgments}

We thank H. Fukiage, A. Banno, M. Sato, A. Kobayashi, A. Fujii, K. Araki, A. Ito, T. Goto, M. Kawabata, M. Iyobe, T. Mizuno, T. Kodera, Y. Nomura, Y. Futaki, T. Yoshihara, and N. Ide for technical assistance and A. Iyama for clerical assistance. This work was supported by JSPS KAKENHI (18604010 and 21591375 to H. Yoshida; 24591474 and 22790949 to T. Yasuda) and by RIKEN Strategic Programs for R\&D (K90-88110).

Address correspondence to: Hisahiro Yoshida, 1-7-22 Suehiro-cho, Tsurumi-ku, Yokohama, Kanagawa 230-0045, Japan. Phone: 81.45.503.7058; E-mail: hisahiro@riken.jp.
1. Jakasa I, et al. Skin barrier function in healthy subjects and patients with atopic dermatitis in relation to filaggrin loss-of-function mutations. J Invest Dermatol. 2011;131(2):540-542.

2. Proksch E, Brandner JM, Jensen JM. The skin: an indispensable barrier. Exp Dermatol. 2008;17(12):1063-1072.

3. Tran QT, et al. EGFR regulation of epidermal barrier function. Physiol Genomics. 2012;44(8):455-469.

4. Franzke CW, et al. Epidermal ADAM17 maintains the skin barrier by regulating EGFR ligand-dependent terminal keratinocyte differentiation. J Exp Med. 2012;209(6):1105-1119.

5. Wang XP, et al. The interleukin- 6 cytokine system regulates epidermal permeability barrier homeostasis. J Invest Dermatol. 2004;123(1):124-131.

6. Oyoshi MK, He R, Kumar L, Yoon J, Geha RS. Cellular and molecular mechanisms in atopic dermatitis. Adv Immunol. 2009;102:135-226.
7. Cavani A, Pennino D, Eyerich K. Th17 and Th22 in skin allergy. Chem Immunol Allergy. 2012;96:39-44.

8. Hao JQ. Targeting interleukin-22 in psoriasis. Inflammation. 2014;37(1):94-99.

9. Sakuraba Y, et al. Molecular characterization of ENU mouse mutagenesis and archives. Biochem Biophys Res Commun. 2005;336(2):609-616.

10. Murray PJ. The JAK-STAT signaling pathway: input and output integration. JImmunol. 2007;178(5):2623-2629.

11. Stark GR, Darnell JE Jr. The JAK-STAT pathway at twenty. Immunity. 2012;36(4):503-514.

12. Matsuda H, et al. Development of atopic dermatitis-like skin lesion with $\operatorname{IgE}$ hyperproduction in NC/Nga mice. Int Immunol. 1997;9(3):461-466.

13. Inoue $\mathrm{M}$, et al. A series of maturity onset diabetes of the young, type 2 (MODY2) mouse models generated by a large-scale ENU mutagenesis program.
Hum Mol Genet. 2004;13(11):1147-1157.

14. Ohtani T, et al. Dissection of signaling cascades through gp130 in vivo: reciprocal roles for STAT3- and SHP2-mediated signals in immune responses. Immunity. 2000;12(1):95-105.

15. Ghoreschi K, Laurence A, O'Shea JJ. Janus kinases in immune cell signaling. Immunol Rev. 2009;228(1):273-287.

16. Mombaerts P, Iacomini J, Johnson RS, Herrup K, Tonegawa S, Papaioannou VE. RAG-1-deficient mice have no mature B and T lymphocytes. Cell. 1992;68(5):869-877.

17. Theoharides TC, et al. Mast cells and inflammation. Biochim Biophys Acta. 2012;1822(1):21-33.

18. Yamazaki M, et al. C-kit gene is expressed by skin mast cells in embryos but not in puppies of Wsh/ Wsh mice: age-dependent abolishment of c-kit gene expression. Blood. 1994;83(12):3509-3516.

19. Rosado C, Pinto P, Rodrigues LM. Comparative 
assessment of the performance of two generations of Tewameter: TM210 and TM300. Int J Cosmet Sci. 2005;27(4):237-241.

20. Man MQ, et al. Deficiency of PPARbeta/delta in the epidermis results in defective cutaneous permeability barrier homeostasis and increased inflammation. JInvest Dermatol. 2008;128(2):370-377.

21. Briot A, et al. Kallikrein 5 induces atopic dermatitis-like lesions through PAR2-mediated thymic stromal lymphopoietin expression in Netherton syndrome. J Exp Med. 2009;206(5):1135-1147.

22. Debela M, et al. Structures and specificity of the human kallikrein-related peptidases KLK 4, 5, 6, and 7. Biol Chem. 2008;389(6):623-632.

23. Komatsu N, et al. Human tissue kallikrein expression in the stratum corneum and serum of atopic dermatitis patients. Exp Dermatol. 2007;16(6):513-519.

24. Li W, et al. The serine protease marapsin is expressed in stratified squamous epithelia and is up-regulated in the hyperproliferative epidermis of psoriasis and regenerating wounds. J Biol Chem. 2009;284(1):218-228.

25. Rodig SJ, et al. Disruption of the Jak1 gene demonstrates obligatory and nonredundant roles of the Jaks in cytokine-induced biologic responses. Cell. 1998;93(3):373-383.

26. Descargues P, et al. Corneodesmosomal cadherins are preferential targets of stratum corneum trypsin- and chymotrypsin-like hyperactivity in Netherton syndrome. J Invest Dermatol. 2006;126(7):1622-1632.

27. Eissa A, Diamandis EP. Human tissue kallikreins as promiscuous modulators of homeostatic skin barrier functions. Biol Chem. 2008;389(6):669-680.

28. Ny A, Egelrud T. Transgenic mice over-expressing a serine protease in the skin: evidence of inter- feron $\gamma$-independent MHC II expression by epidermal keratinocytes. Acta Derm Venereol. 2003;83(5):322-327.

29. Hsu L, Armstrong AW. JAK inhibitors: treatment efficacy and safety profile in patients with psoriasis. JImmunol Res. 2014;2014:283617.

30. Clark JD, Flanagan ME, Telliez JB. Discovery and development of Janus kinase (JAK) inhibitors for inflammatory diseases. J Med Chem. 2014;57(12):5023-5038.

31. Nakajima K, et al. Distinct roles of IL-23 and IL-17 in the development of psoriasis-like lesions in a mouse model. JImmunol. 2011;186(7):4481-4489.

32. Yang XP, et al. Opposing regulation of the locus encoding IL-17 through direct, reciprocal actions of STAT3 and STAT5. Nat Immunol. 2011;12(3):247-254.

33. Shibata S, et al. IL-27 activates Th1-mediated responses in imiquimod-induced psoriasis-like skin lesions. JInvest Dermatol. 2013;133(2):479-488.

34. Wolk K, et al. IL-22 and IL-20 are key mediators of the epidermal alterations in psoriasis while IL-17 and IFN-gamma are not. JMol Med (Berl). 2009;87(5):523-536.

35. Sabat R, Wolk K. Research in practice: IL-22 and IL-20: significance for epithelial homeostasis and psoriasis pathogenesis. J Dtsch Dermatol Ges. 2011;9(7):518-523.

36. Dong X, Chen J, Zhang Y, Cen Y. Mast cell chymase promotes cell proliferation and expression of certain cytokines in a dose-dependent manner. Mol Med Rep. 2012;5(6):1487-1490.

37. Harper EG, et al. Th17 cytokines stimulate CCL20 expression in keratinocytes in vitro and in vivo: implications for psoriasis pathogenesis. J Invest Dermatol. 2009;129(9):2175-2183.

38. Wollenberg A, Rawer HC, Schauber J. Innate immunity in atopic dermatitis. Clin Rev Allergy
Immunol. 2011;41(3):272-281.

39. Horimukai K, et al. Application of moisturizer to neonates prevents development of atopic dermatitis. J Allergy Clin Immunol. 2014;134(4):824-830 e826.

40. Simpson EL, et al. Emollient enhancement of the skin barrier from birth offers effective atopic dermatitis prevention. J Allergy Clin Immunol. 2014;134(4):818-823.

41. Sabrautzki S, et al. An ENU mutagenesis-derived mouse model with a dominant Jak1 mutation resembling phenotypes of systemic autoimmune disease. Am J Pathol. 2013;183(2):352-368.

42. O'Shea JJ, Plenge R. JAK and STAT signaling molecules in immunoregulation and immune-mediated disease. Immunity. 2012;36(4):542-550.

43. Flex E, et al. Somatically acquired JAK1 mutations in adult acute lymphoblastic leukemia. JExp Med. 2008;205(4):751-758.

44. Porcu M, Gielen O, Cools J, De Keersmaecker K. JAK1 mutation analysis in T-cell acute lymphoblastic leukemia cell lines. Haematologica. 2009;94(3):435-437.

45. Casanova JL, Holland SM, Notarangelo LD. Inborn errors of human JAKs and STATs. Immunity. 2012;36(4):515-528.

46. Zhang J, et al. The genetic basis of early T-cell precursor acute lymphoblastic leukaemia. Nature. 2012;481(7380):157-163.

47. Yoshida Y, et al. PosMed (Positional Medline): prioritizing genes with an artificial neural network comprising medical documents to accelerate positional cloning. Nucleic Acids Res. 2009;37(Web Server issue):W147-W152.

48. Horvath CM, Wen Z, Darnell JE Jr. A STAT protein domain that determines DNA sequence recognition suggests a novel DNA-binding domain. Genes Dev. 1995;9(8):984-994. 